\title{
Dietary Emulsifiers Alter Composition and Activity of the Human Gut Microbiota in vitro, Irrespective of Chemical or Natural Emulsifier Origin
}

\author{
Lisa Miclotte ${ }^{1}$, Kim De Paepe ${ }^{1}$, Leen Rymenans ${ }^{2}$, Chris Callewaert', Jeroen Raes ${ }^{2}$, \\ Andreja Rajkovic ${ }^{3}$, John Van Camp ${ }^{3}$ and Tom Van de Wiele ${ }^{1 *}$
}

${ }^{1}$ Center for Microbial Ecology and Technology (CMET), Ghent University, Ghent, Belgium, ${ }^{2}$ Vlaams Instituut voor Biotechnologie (VIB) Nucleomics Core, Lab of Molecular Bacteriology - Rega, KULeuven, Ghent, Belgium, ${ }^{3}$ Department of Food Technology, Food Safety and Health, Ghent University, Ghent, Belgium

OPEN ACCESS

Edited by:

Francesca Turroni,

University of Parma, Italy

Reviewed by:

María Esteban-Torres,

University College Cork, Ireland

Sandrine Ellero-Simatos,

Institut National de la Recherche

Agronomique (INRA), France

*Correspondence:

Tom Van de Wiele

tom.vandewiele@ugent.be

Specialty section:

This article was submitted to

Microbial Symbioses,

a section of the journal

Frontiers in Microbiology

Received: 29 June 2020

Accepted: 09 October 2020

Published: 05 November 2020

Citation:

Miclotte L, De Paepe K, Rymenans L, Callewaert C, Raes J, Rajkovic A, Van Camp J and Van de Wiele $T$ (2020) Dietary Emulsifiers Alter Composition and Activity of the Human Gut Microbiota in vitro,

Irrespective of Chemical or Natural Emulsifier Origin.

Front. Microbiol. 11:577474.

doi: 10.3389/fmicb.2020.577474
The use of additives in food products has become an important public health concern. In recent reports, dietary emulsifiers have been shown to affect the gut microbiota, contributing to a pro-inflammatory phenotype and metabolic syndrome. So far, it is not yet known whether similar microbiome shifts are observable for a more diverse set of emulsifier types and to what extent these effects vary with the unique features of an individual's microbiome. To bridge this gap, we investigated the effect of five dietary emulsifiers on the fecal microbiota from 10 human individuals upon a $48 \mathrm{~h}$ exposure. Community structure was assessed with quantitative microbial profiling, functionality was evaluated by measuring fermentation metabolites, and pro-inflammatory properties were assessed with the phylogenetic prediction algorithm PICRUSt, together with a TLR5 reporter cell assay for flagellin. A comparison was made between two mainstream chemical emulsifiers (carboxymethylcellulose and P80), a natural extract (soy lecithin), and biotechnological emulsifiers (sophorolipids and rhamnolipids). While fecal microbiota responded in a donor-dependent manner to the different emulsifiers, profound differences between emulsifiers were observed. Rhamnolipids, sophorolipids, and soy lecithin eliminated $91 \pm 0,89 \pm 1$, and $87 \pm 1 \%$ of the viable bacterial population after $48 \mathrm{~h}$, yet they all selectively increased the proportional abundance of putative pathogens. Moreover, profound shifts in butyrate $(-96 \pm 6,-73 \pm 24$, and $-34 \pm 25 \%)$ and propionate $(+13 \pm 24$, $+88 \pm 50$, and $+29 \pm 16 \%)$ production were observed for these emulsifiers. Phylogenetic prediction indicated higher motility, which was, however, not confirmed by increased flagellin levels using the TLR5 reporter cell assay. We conclude that dietary emulsifiers can severely impact the gut microbiota, and this seems to be proportional to their emulsifying strength, rather than emulsifier type or origin. As biotechnological emulsifiers were especially more impactful than chemical emulsifiers, caution is warranted when considering them as more natural alternatives for clean label strategies.

Keywords: dietary emulsifiers, human gut microbiota, interindividual variablility, microbiome composition, emulsifier origin, microbiome functionality 


\section{INTRODUCTION}

The current obesity crisis and related health conditions are increasingly associated with the overconsumption of so-called ultra-processed food products (Broussard and Devkota, 2016; Monteiro et al., 2017; Rauber et al., 2018; Waterlander et al., 2018; Branca et al., 2019). Food additives are characteristic elements of said products (Monteiro et al., 2013, 2017; Carocho et al., 2014) and are added to enhance, among others, shelf life, palatablility, texture, color, and nutritional value. However, the health impact of certain food additives has always been questioned (Payne et al., 2012; Carocho et al., 2014; Miclotte and Van de Wiele, 2019), and at this moment, the use of food additives in food products is one of the main public concerns about food in Europe (EFSA, 2019).

Diet is known to have a strong and fast impact on the gut microbiota (Musso et al., 2010; Martínez Steele et al., 2017; Ding et al., 2019), which is generally considered an important parameter of gut and overall health (Musso et al., 2010; Bischoff, 2011; Ding et al., 2019). An unbalanced gut microbiota is being related to several physical and mental illnesses and conditions (Ding et al., 2019). With respect to obesity and non-communicable diseases, a dysbiosed gut microbiota is characterized by a lower alpha diversity and is related to increased harvest from food and decreased fatty acid oxidation, glucose tolerance, production of satiety hormones, and intestinal barrier integrity (Musso et al., 2010).

Recently, research has emerged that ties the consumption of additives to health markers through the gut microbiota. Dietary emulsifiers in particular have been proposed to display a destabilizing impact on gut health. Chassaing et al. $(2015,2017)$ found in vivo that sodium carboxymethylcellulose (CMC) and polysorbate 80 (P80) increase gut microbial motility and lower mucus layer thickness, yielding an increased production of pro-inflammatory compounds, low-grade gut inflammation, and weight gain. Another study has linked glycerol monolaurate (GML) with signatures of metabolic syndrome together with the alterations of gut microbiota composition, among which decreased abundance of Akkermansia muciniphila and increased abundance of Escherichia coli (Jiang et al., 2018). The latter study is particularly relevant since GML is one of the World's most widely used dietary emulsifiers (E471).

Knowledge that is currently still lacking from literature regarding the impact of dietary emulsifiers is what characteristics of an emulsifier determine its destabilizing effects, whether alternative, more natural emulsifiers could be safer and to what extent the unique features of an individual's microbiome play a role in the purported effects on the microbiome.

The present study describes the effects of five dietary emulsifiers: CMC, P80, soy lecithin, sophorolipids, and rhamnolipids (RLs). The first two, CMC and P80 are the synthetic emulsifiers that have been used for years and both are considered safe for human oral consumption. CMC is a water soluble anionic polymer with water-binding properties, due to which it is used as a thickener, emulsifier, or water-retainer in applications like pharmaceuticals, food products, paper, cosmetics, detergents, etc. (Hercules Inc., and Aqualon, 1999; Biswal and Singh, 2004). In Europe, CMC can be used in many food products at quantum satis levels (European Commision, 2014), and in the United States, CMC carries the GRAS-status (generally recognized as safe) for the applications in food (FDA, 2019b, 2020). P80 is a member of the polysorbates, a group of non-ionic surfactants with applications mainly in the food, cosmetics, and pharmaceutical industries (Nielsen et al., 2016; FMI, 2020). With an acceptable daily intake of $25 \mathrm{mg} / \mathrm{kg}$ BW/day (Aguilar et al., 2015), EFSA allows its use in products like sauces, soups, chewing gum, coconut milk, dairy products, and usually at maximal concentrations of $10-10,000 \mathrm{mg} / \mathrm{kg}$, depending on the product (European Commision, 2011). Also the United States Food and Drug Administration (FDA) limits the use of P80 to 4-10,000 mg/ $\mathrm{kg}$, depending on the product (FDA, 2019a).

Soy lecithin is a mixture of phospholipids (at least 60\%), triglycerides, sterols, and carbohydrates obtained by extraction from soybeans. It is more widely used than CMC and P80, primarily in bakery products, ice creams, chocolate etc. (European Commision, 2018). Lecithins are allowed by EFSA in most food applications in quantum satis levels, and also the FDA considers soy lecithin a GRAS compound (Carocho et al., 2014; FDA, 2019c). Even though soy lecithin is considered safe or even beneficial for health (Ehehalt et al., 2010; Mourad et al., 2010; Stremmel et al., 2010), the impact of soy lecithin on the gut microbiota has never been studied. Since this compound is one of the most extensively used food emulsifiers, it was incorporated in this research.

Finally, rhamnolipids and sophorolipids are two biotechnological emulsifiers of microbial origin. Due to their advantageous properties with respect to (eco) toxicity and waste stream reuse (Haba et al., 2003; Van Bogaert et al., 2011; Costa et al., 2017), they are currently under consideration as novel food additives (Cameotra and Makkar, 2004; Costa et al., 2017; Nitschke and Silva, 2018). Their strong emulsifying capacities (Van Bogaert et al., 2011; Costa et al., 2017) and more natural origin (biotechnological production from renewable resources) could qualify them as adequate alternatives for emulsifiers of chemical origin, which the food industry is currently seeking to replace under the umbrella of the "clean label" trend (Asioli et al., 2017; Costa et al., 2017; Nitschke and Silva, 2018). However, given their strong antimicrobial properties, an evaluation at the level of the gut microbiota is highly warranted before such applications can be legalized.

Here, we investigated the effects of the five above-mentioned dietary emulsifiers on human fecal microbiota through $48 \mathrm{~h}$ in vitro batch incubations. This set of emulsifiers enables the comparison of the previously-studied chemical emulsifiers with the natural extract lecithin and with biosurfactants. In order to take into account interindividual variability in microbiome composition as a possible determinant of the putative impact from emulsifiers, we separately assessed microbial incubations from 10 different individuals.

\section{MATERIALS AND METHODS}

\section{Experimental Design}

Fecal material from 10 human individuals was collected and separately incubated for $48 \mathrm{~h}$ with the five emulsifiers at three 
concentrations $[0.005,0.05$, and $0.5 \% \quad(\mathrm{~m} / \mathrm{v})]$. Emulsifier concentrations were chosen based on the maximal legal concentration in food products (EFSA and FDA), which comply with commonly applied concentrations in food products (Mallet, 1992; Adams et al., 2004; Msagati, 2012). Each donor incubation series also included a control condition, in which a sham treatment with an equivalent volume of distilled water was performed.

The emulsifiers used during this study were sodium CMC, P80, soy lecithin, sophorolipids, and rhamnolipids. CMC (catalog number 419303: average molecular weight of 250,000 g/mol and degree of substitution of 0.9), P80 (P4780 - suitable for cell culture) and rhamnolipids (RLs: R90-90\% pure) were obtained from Sigma Aldrich, St. Louis, MO, United States. Soy lecithin was obtained from Barentz Unilecithin (UNILECISL non GMO IP), and sophorolipids were obtained from the UGent Inbio group from the Centre for Synthetic Biology. The latter were described as $75 \%(\mathrm{w} / \mathrm{v})$ solutions, and their composition was determined to be mainly lactonic, diacetylated C18:1 SL.

Donors 2 and 6 reported to follow a vegetarian and vegan diet. All other donors consumed an omnivorous diet. The age of the four female and six male donors varied from 23 to 53 years old. None of the donors received any antibiotic treatment in the 3 months prior to their donation. Experimental work with fecal microbiota from human origin was approved by the Ethical Committee of the Ghent University hospital under the registration number BE670201836318.

\section{Batch Incubation}

Before incubation, the five emulsifiers were supplemented to amber penicillin bottles containing $40 \mathrm{ml}$ of autoclaved low-sugar nutritional medium (per L: $0.25 \mathrm{~g}$ gum arabic, $0.5 \mathrm{~g}$ pectin, $0.25 \mathrm{~g}$ xylan, $1 \mathrm{~g}$ starch, $3 \mathrm{~g}$ yeast extract, $1 \mathrm{~g}$ proteose peptone, and $2 \mathrm{~g}$ pig gastric mucin; all from Sigma Aldrich, St. Louis, $\mathrm{MO})$. The amounts of emulsifiers to add were calculated to obtain concentrations of $0.005,0.05$, and $0.5 \%(\mathrm{~m} / \mathrm{v})$ in a final volume of $50 \mathrm{ml}$ (the volume obtained after addition of the fecal slurry). The bottles were stored in a $4^{\circ} \mathrm{C}$ fridge until use (for maximum 3 days).

At the start of the batch experiments, the penicillin flasks containing nutritional medium and emulsifiers were brought to room temperature to provide an ideal growth environment for the fecal bacteria. Fresh fecal samples were then collected in airtight plastic lidded containers. AnaeroGen ${ }^{\mathrm{TM}}$ sachets (Oxoid Ltd., Basingstoke, Hampshire, UK) were used to sequester $\mathrm{O}_{2}$. The samples were stored at $4^{\circ} \mathrm{C}$ until the use for a maximum of $3 \mathrm{~h}$. A fecal inoculum was then prepared as described in De Boever et al. (2000), by mixing 20\% (w/v) fecal material into a $0.1 \mathrm{M}$ anaerobic phosphate buffer ( $\mathrm{pH}$ 6.8) supplemented with $1 \mathrm{~g} / \mathrm{L}$ sodium thioglycanate as a reducing agent. Into each penicillin bottle, $10 \mathrm{ml}$ of fecal inoculum was added, after which the bottles were closed with butyl rubber stoppers and aluminum caps. The headspaces were flushed with a $\mathrm{N}_{2} / \mathrm{CO}_{2}$ (80/20)-gas mixture using a gas exchange equipment to obtain anaerobic conditions and incubated in an IKA ${ }^{\circledR} \mathrm{KS}$ $4000 \mathrm{I}$ Control shaker at $200 \mathrm{rpm}$ at $37^{\circ} \mathrm{C}$. During the course of the experiments, the $\mathrm{pH}$ was followed up every day with a Prosense QP108X pH-electrode connected to a Consort C3020 multi parameter analyzer to ensure stable and viable growth conditions ( $\mathrm{pH}$ remained within 5.5-6.8).

Aliquots were taken on three timepoints: immediately after the start of the incubation (T0; 2-3 h after combining the fecal inoculum with the medium containing emulsifier), after $24 \mathrm{~h}$ of incubation (T1) and after $48 \mathrm{~h}$ of incubation (T2). Samples were taken for short chain fatty acid (SCFA) analysis $(1 \mathrm{ml}), 16 \mathrm{~S}$ rRNA gene amplicon sequencing $(1 \mathrm{ml})$, for flagellin detection $(500 \mu \mathrm{l})$, and for immediate fluorescent cell staining and flow cytometry $(100 \mu \mathrm{l})$. SCFA-, flagellin-, and sequencingsamples were stored at $-20^{\circ} \mathrm{C}$ until analysis.

\section{Intact/Damaged Cell Counts}

To assess the impact of the emulsifiers on total and intact cell concentrations, cell staining with $\mathrm{SYBR}^{\circledR}$ green and propidium iodide was performed after which the cells were counted on an Accuri C6+ Flow cytometer from BD Biosciences Europe. The combination of these two cell stains is frequently used to distinguish intact bacterial cells from cells damaged at the level of the cell membrane, since $\mathrm{SYBR}^{\circledR}$ green enters any cell rapidly, while propidium iodide, being a larger molecule, enters intact cells much slower and mainly stains damaged cells within commonly applied incubation times (Van Nevel et al., 2013). Samples were analyzed immediately after sampling to preserve the intact cell community. Dilutions up to $10^{-4}$ and $10^{-5}$ were prepared in 96-well plates using $0.22 \mu \mathrm{m}$ filtered $0.01 \mathrm{M}$ phosphate buffered saline (PBS; $\mathrm{HPO}_{4}{ }^{2-} / \mathrm{H}_{2} \mathrm{PO}_{4}^{-}, 0.0027 \mathrm{M} \mathrm{KCl}$, and $0.137 \mathrm{M} \mathrm{NaCl}, \mathrm{pH} \mathrm{7.4,}$ at $25^{\circ} \mathrm{C}$ ), and these were subsequently stained with $\mathrm{SYBR}^{\circledR}$ green combined with propidium iodide (SGPI, 100 $\times$ concentrate SYBR $^{\circledR}$ Green I, Invitrogen, and $50 \times 20 \mathrm{mM}$ propidium iodide, InvitroGen, in $0.22 \mu \mathrm{m}$-filtered dimethyl sulfoxide; Van Nevel et al., 2013; Props et al., 2016). After 25 min of incubation, the intact and damaged cell populations were measured immediately with the flow cytometer, which was equipped with four fluorescence detectors (530/30, 585/40, $>670$, and $675 / 25 \mathrm{~nm}$ ), two scatter detectors and a $20 \mathrm{~mW}$ $488 \mathrm{~nm}$ laser. The flow cytometer was operated with Milli-Q (Merck Millipore, Belgium) as sheath fluid. The blue laser $(488 \mathrm{~nm})$ was used for the excitation of the stains, and a minimum of 10,000 cells per sample were measured for accurate quantification. Settings used were an FLH-1 limit of 1,000 , a measurement volume of $25 \mu \mathrm{l}$, and the measurement speed was set to "fast." Cell counts were obtained by gating the intact and damaged cell populations in $\mathrm{R}$ (version 3.6.2) according to the Phenoflow-package (v1.1.6; Props et al., 2016). Gates were verified using data from negative control samples (only $0.22 \mu \mathrm{m}$ filtered $0.01 \mathrm{M}$ PBS; Figure S1 in Supplementary Data Sheet 1).

\section{SCFA-Analysis}

The SCFA-concentrations were determined by means of diethyl ether extraction and capillary gas chromatography coupled to a flame ionization detector as described by De Paepe et al. (2017) and Anderson et al. (2017). Briefly, $1 \mathrm{ml}$ aliquots were diluted 
$2 \times$ with $1 \mathrm{ml}$ milli-Q water, and SCFA were extracted by adding approximately $400 \mathrm{mg} \mathrm{NaCl}, 0.5 \mathrm{ml}$ concentrated $\mathrm{H}_{2} \mathrm{SO}_{4}$, $400 \mu \mathrm{l}$ of 2-methyl hexanoic acid internal standard and $2 \mathrm{ml}$ of diethyl ether before mixing for $2 \mathrm{~min}$ in a rotator and centrifuging at 3,000 $\mathrm{g}$ for $3 \mathrm{~min}$. Upper layers were collected and measured using a GC-2014 capillary gas chromatograph (Shimadzu, Hertogenbosch, Netherlands), equipped with a capillary fatty acid-free EC-1000 Econo-Cap column (Alltech, Lexington, KY, United States), $25 \mathrm{~m} \times 0.53 \mathrm{~mm}$; film thickness $1.2 \mu \mathrm{m}$, and coupled to a flame ionization detector and split injector. One sample [donor 9, timepoint 2-0.05\% (m/v) $\mathrm{CMC}$ ] returned only zero values, presumably due to a technical error. This sample was therefore omitted prior to computational analyses.

\section{Amplicon Sequencing}

Samples from T0 and T2 were selected for Illumina $16 \mathrm{~S}$ rRNA gene amplicon sequencing. The samples $(1 \mathrm{ml})$ were first centrifuged for $5 \mathrm{~min}$ at $30,130 \mathrm{~g}$ in an Eppendorf 5430 $\mathrm{R}$ centrifuge to obtain a cell pellet. After removing the supernatant, the pellets were subjected to DNA-extraction (Vilchez-Vargas et al., 2013; De Paepe et al., 2017). The pellets were dissolved in $1 \mathrm{ml}$ Tris $/ \mathrm{HCl}(100 \mathrm{mM}, \mathrm{pH}=8.0)$ supplemented with $100 \mathrm{mM}$ EDTA, $100 \mathrm{mM} \mathrm{NaCl}, 1 \%$ (w/v) polyvinylpyrrolidone, and $2 \%(\mathrm{w} / \mathrm{v})$ sodium dodecyl sulfate after which $200 \mathrm{mg}$ glass beads $(0.11 \mathrm{~mm}$ Sartorius, Gottingen, Germany) were added, and the cells were lysed for $5 \mathrm{~min}$ at 2,000 rpm in a FastPrep VR-96 instrument (MP Biomedicals, Santa Ana, CA). The beads were then precipitated by centrifugation for $5 \mathrm{~min}$ at $30,130 \mathrm{~g}$, and the supernatant was collected. Purification of DNA took place by the extraction of cellular proteins with $500 \mu \mathrm{l}$ phenol-chloroform-isoamilyc alcohol $25-24-1$ at $\mathrm{pH}$ and $700 \mu \mathrm{l} 100 \%$ chloroform. The DNA was precipitated by adding 1 volume of ice-cold isopropyl alcohol and $45 \mu \mathrm{l}$ sodium acetate and cooling for at least $1 \mathrm{~h}$ at $-20^{\circ} \mathrm{C}$. Isopropyl alcohol was then separated from DNA by centrifugation for $30 \mathrm{~min}$ at $4^{\circ} \mathrm{C}$ and at $30,130 \mathrm{~g}$, and the pellet was dried by pouring off the supernatant. It was resuspended in $100 \mathrm{ml} 1 \times$ TE buffer $(10 \mathrm{mM}$ Tris, $1 \mathrm{mM}$ EDTA) for storage at $-20^{\circ} \mathrm{C}$.

DNA-quality was verified by electrophoresis in a $1.5 \%$ $(\mathrm{w} / \mathrm{v})$ agarose gel (Life technologies, Madrid, Spain), and DNA-concentration was determined using a QuantiFluor ${ }^{\circledR}$ dsDNA kit (detection limit: 50 pg/ml; sensitivity: 0.01-200 ng/ $\mu \mathrm{l})$ and $\mathrm{GloMax}^{\circledR}$-Multi+ system (Promega, Madison, WI) with the blue fluorescence optical kit installed (Ex: $490 \mathrm{~nm}$ and $\mathrm{Em}$ : $510-570 \mathrm{~nm}$ ).

Library preparation and next generation 16S rRNA gene amplicon sequencing were performed at the VIB nucleomics core (VIB, Gasthuisberg Campus, Leuven, Belgium) as described in Tito et al. (2017). The V4 region of the $16 \mathrm{~S}$ rRNA gene was amplified using the bacterial 515F (GTGYCAGCMGCCGCGGTAA) and the 806R (GGACTACNVGGGTWTCTAAT) primers, which were modified with both Illumina adapters as well as adapters for directional sequencing. Sequencing was then performed on an Illumina MiSeq platform (Illumina, Hayward, CA, United States) according to manufacturer's guidelines.
One sample [donor 3, timepoint 2, 0.05\% (m/v) sophorolipids] failed to sequence. The sequencing data have been submitted to the National Center for Biotechnology Information (NCBI) database under the accession number PRJNA630547.

Processing of amplicon data was carried out using mothur software version 1.40 .5 and guidelines (Kozich et al., 2013). First, contigs were assembled, resulting in 14,977,727 sequences, and ambiguous base calls were removed. Sequences with a length of 291 or 292 nucleotides were then aligned to the silva_seed nr.123 database, trimmed between positions 11,895 and 25,318 (Quast et al., 2013). After removing the sequences containing homopolymers longer than nine base pairs, 92\% of the sequences were retained resulting in 2,957,626 unique sequences. A pre-clustering step was then performed, allowing only three differences between sequences clustered together and chimera. vsearch was used to remove chimeras, retaining $79 \%$ of the sequences. The sequences were then classified using a naïve Bayesian classifier against the Ribosomal Database Project (RDP) 16S rRNA gene training set version 16, with a cut-off of $85 \%$ for the pseudobootstrap confidence score. Sequences that were classified as Archaea, Eukaryota, Chloroplasts, unknown, or Mitochondria at the kingdom level were removed. Finally, sequences were split at the order level into taxonomic groups using the OptiClust method with a cut-off of 0.03 . The data were classified at a $3 \%$ dissimilarity level into OTUs resulting in a shared (count table) and a tax file (taxonomic classification).

For the entire dataset of 319 samples, 95,511 OTUs were detected in 175 genera. An OTU was in this manuscript defined as a collection of sequences with a length between 291 and 292 nucleotides and with $97 \%$ or more similarity to each other in the V4 region of their $16 \mathrm{~S}$ rRNA gene after applying hierarchical clustering.

\section{Cell Culture for Flagellin Detection}

Murine TLR5-expressing HEK 293 cells (InvivoGen), which are designed to respond to bacterial flagellin in cell culture medium, were cultured according to the manufacturer's guidelines. Cells were grown from an in house created frozen stock in Dulbecco's modified Eagle's growth medium (DMEM; $4.5 \mathrm{~g} / \mathrm{L}$ glucose, $10 \%$ fetal bovine serum, $50 \mathrm{U} / \mathrm{ml}$ penicillin, $50 \mu \mathrm{g} / \mathrm{ml}$ streptomycin, and $2 \mathrm{mM}$ L-glutamine) supplemented with $100 \mu \mathrm{g} / \mathrm{ml} \mathrm{Normocin}{ }^{\mathrm{TM}}$ and maintained in culture in DMEM growth medium supplemented with $100 \mu \mathrm{g} / \mathrm{ml}$ Normocin $^{\mathrm{TM}}, 10 \mu \mathrm{g} / \mathrm{ml}$ of blasticidin, and $100 \mu \mathrm{g} / \mathrm{ml}$ of Zeocin ${ }^{\mathrm{TM}}$. Medium was refreshed every 2 days, and the cells were passaged when reaching $70-80 \%$ confluency.

Assays for flagellin detection were performed as instructed by InvivoGen, using cells from the passages $4-9$. Samples from donors 3, 5, and 7 were selected for this assay based on a high, intermediate, and low metabolic responses to the emulsifiers, as measured by the SCFA-levels. Before combining with the HEK-blue cells, the samples were purified to obtain only the bacterial cells by first diluting them one-fourth in UltraPure ${ }^{\mathrm{TM}}$ DNase/RNase-Free distilled water (InvitroGen), then centrifuging twice at $4,226 \mathrm{~g}$ for $10 \mathrm{~min}$, with a washing step using $0.22 \mu \mathrm{m}$ filtered PBS in between. The resulting cell pellet was dissolved 
into $0.22 \mu \mathrm{m}$ filtered PBS. A standard curve $(1.25-1.95 \mathrm{ng} / \mathrm{ml})$, prepared from recombinant flagellin from Salmonella typhymurium (RecFLA-ST, InvivoGen) in sterile water was also added to the plate in triplicate. After an incubation for $23 \mathrm{~h}$, absorbances were obtained using a Tecan Infinite F50 plate reader at $620 \mathrm{~nm}$.

As a check for the viability of the cell culture after combination with the samples, a resazurin assay was performed. To this end, the supernatant from the cell culture plate used for the flagellin assay was discarded after the first incubation phase. The cells were then washed using $0.22 \mu \mathrm{m}$ filtered PBS. For the detection part of the assay, three wells were spiked with $20 \mu \mathrm{l}$ of a $5 \%$ Trition solution, as a positive control, while the rest of the wells received $20 \mu \mathrm{l}$ PBS. Then, $180 \mu \mathrm{l}$ of a $0.01 \mu \mathrm{g} / \mathrm{ml}$ resazurin solution was added to all wells. After $3 \mathrm{~h}$ of incubation at $37^{\circ} \mathrm{C}$ and $10 \% \mathrm{CO}_{2}$, cell activity was measured using a Glomax ${ }^{\circledR}$ Multi1 system (Promega, Madison, WI) with filter the green fluorescence optical kit (Ex: $525 \mathrm{~nm}$ and Em: 580-640 nm).

\section{Data Analysis and Statistics}

Data visualization and processing was performed in $\mathrm{R}$ version 3.4.2 (2017-09-28; R Core Team, 2016) and Excel 2016. All hypothesis testing was done based on a significance level of $5 \%(\alpha=0.05)$.

\section{Cell Counts and SCFA}

After loading the cell count table in $\mathrm{R}$, total cell counts were calculated as the sum of the intact and damaged cell counts. The data were explored by calculating intact/damaged cell count ratio's and percentages of intact cells at different timepoints. For plotting, both the $10,000 \times$ and $100,000 \times$ dilutions were taken into account. Boxplots of total and intact cell counts, as well as intact/damaged ratio's were created using ggplot2 (v3.2.1) in which the stat_compare_means function was used to check the significance of the emulsifier effect, by means of a Kruskal-Wallis test.

Statistical analysis of SCFA-levels was similar to that of the cell counts. Production levels of acetate, propionate, and butyrate over $48 \mathrm{~h}\left(\mathrm{C}_{\mathrm{T} 2}-\mathrm{C}_{\mathrm{T} 0}\right)$ were first calculated, and then the boxplots were created using ggplot2. Significance of the effect of the emulsifier treatment was tested with stat_compare_means using Wilcoxon Rank Sum tests with Holm's correction and KruskalWallis test for overall group comparison.

\section{Amplicon Sequencing Data}

The shared and taxonomy files resulting from the mothur pipeline were loaded into R for further processing. Singletons (OTUs occurring only once over all samples) were removed, resulting in 36,496 OTUs being retained (McMurdie and Holmes, 2014). Rarefaction curves were created to evaluate the sequencing depth (Figure S2 in Supplementary Data Sheet 1; Oksanen et al., 2019). As the number of $16 \mathrm{~S}$ rRNA gene copies present within bacteria differs between species, a copy number correction of the reads was carried out by first classifying the representative sequences of the OTUs (also obtained via the mothur pipeline) using the online RDP classifier tool, then obtaining both a copy number corrected read classification and a non-copy number corrected one, calculating the copy number by dividing both and finally dividing the acquired read counts in the shared file by the calculated copy numbers.

Both relative and absolute abundances of the OTUs and genera were calculated from the copy number corrected read counts and were explored via bar plots using ggplot2 (v3.2.1). Relative abundances were calculated as percentages of the total read counts per sample. Absolute abundances were calculated (quantitative microbial profiling) by multiplying the total cell counts obtained via flow cytometry with the relative abundances of the OTUs (similar to Vandeputte et al., 2017).

Overall community composition was visualized using principle coordinate analysis ( $\mathrm{PcOA})$ on the abundance based Jaccard distance matrix using the cmdscale-function in the stats (v3.6.2) package. To investigate the effects of the individual constraints on the microbial community, a series of distance based redundancy analyses (dbRDAs) was then performed on the scores obtained in the PCoA on the Jaccard distance matrix using the capscale function in the vegan (v2.5.6) package. Permutation tests were used to evaluate the significance of the models and of the explanatory variables (De Paepe et al., 2018). The global model included the factors emulsifier, emulsifier concentration, timepoint, and donor as explanatory variables and the absolute abundances of the genera as explanatory variables. In a first dbRDA, this full model was included, to investigate the share of variance explained by each constraint variable. The timepoint factor was distinguished as the factor causing the largest share of variance, and since its effect was of little interest to us it was partialled out in further dbRDAs. To check for the effect of the donor variable on the microbial community, second and third dbRDAs were performed, with and without conditioning of the donor variable. The final model then visualized the effects of the treatments (defined by factors emulsifier and emulsifier concentration). The results of the dbRDAs were plotted as Type II scaling correlation triplots showing the two first constrained canonical axes (labeled as dbRDA Dim 1/2) and the proportional constrained eigenvalues representing their contribution to the total (both constrained and unconstrained) variance.

The Chao1, ACE-1, Shannon, Simpson, InvSimpson, and Pielou diversity indices were calculated for the microbial community after $48 \mathrm{~h}$ incubation based on the copy number corrected OTU-table using the SPECIES (v1.0) package and the diversity function in the vegan (v2.5.6) package. Indices were plotted using ggplot2, and significances were tested using pairwise Wilcoxon Rank Sum tests with Holm's correction (ggpubr package v.0.2.4).

To evaluate differential abundance of genera between emulsifier treatments and control, the DESeq2 package ( $\mathrm{v}$ 1.24.0) was applied on the copy number corrected count-table at genus level. In order to streamline the DESeq-analysis, pre-filtering according to McMurdie and Holmes (2014) was first applied on the copy number corrected count-table, after which a genuslevel table was created using the aggregate function (stats package v3.6.3). In the generalized linear model, the factor timepoint, donor, and treatment - a concatenation of the 
emulsifiers and their concentrations - were included. A likelihood ratio test was employed within the DESeq function on the reduced model, containing only the factors donor and timepoint, to test for the significance of the model. Low count genera were subjected to an empirical Bayesian correction (Love et al., 2014). For pairwise comparison of treatments versus controls, Wald tests were used after shrinkage of the Log2FoldChange (L2FC) values by means of the lfcShrink function. Values of $p$ were adjusted by means of a Benjamini-Hochberg procedure (Love et al., 2014). Results were visualized in volcanoplots, displaying the $-\log$ (adjusted $p$-value) versus the Log2FoldChange of each genus. Additionally, box plots were created showing the log-transformed pseudocounts extracted by the plotCounts function for each genus that showed significant differential abundance. Since for CMC and P80, no significantly altered genera were found, and these emulsifiers were omitted from the boxplots.

Finally, to summarize relations between the emulsifier treatments and the intact cell counts, the SCFA-data and the 16S rRNA sequencing data, a partial redundancy analysis was carried out performed using the rda function in the vegan package (v2.5.6). The acetate, propionate, and butyrate concentrations, the intact cell counts and the relative abundance of the genera were set as response variables and the factors emulsifier, emulsifier concentration, donor, and timepoint as explanatory variables. Since the response variables carried different units, they were first centered on their mean using the scale function (base $\mathrm{R}$ v3.6.2). The factors donor and timepoint were partialled out to visualize solely the effect of the emulsifier treatments. The statistical significance of the effects was tested via a permutation tests, and the results were plotted in a Type II correlation triplot showing the first two constrained canonical axes (RDA1/2) annotated with their proportional eigenvalues representing their contribution to the constrained variance. The sites were calculated as weighed sums of the scores of the response variables.

\section{Metagenome Prediction}

Indications of functionality from phylogenetic information were obtained using PICRUSt (Phylogenetic Investigation of Communities by Reconstruction of Unobserved States; Langille et al., 2013). An OTU-table was first generated against the Greengenes reference database (v13.8) using a closed ref OTU-picking strategy. The obtained OTU-table was then run through PICRUSt's normalize_by_copy_number.py script (Langille et al., 2013), which divides the abundance of each OTU by its inferred $16 \mathrm{~S}$ copy number (the copy number is inferred from the closest genome representative for a $16 \mathrm{~S}$ Greengenes reference sequence). The metagenome was then predicted using Kyoto Encyclopedia of Genes and Genomes (KEGG) database (Kanehisa et al., 2012). The prediction provided an annotated table of predicted gene family counts for each sample, where gene families were grouped by KEGG orthology identifiers. Significantly different L2-level pathways across emulsifier concentration were visualized in boxplots using the ggplot2 package (v3.2.1). Kruskal-Wallis tests were performed for the overall comparison of emulsifier concentrations within
L2-pathways for each emulsifier and Wilcoxon rank sum tests were used for pairwise comparison of emulsifier concentrations vs. control. Also relying on PICRUSt (Langille et al., 2013), the BugBase tool (Ward et al., 2017) was used to determine the relative degree of biofilm formation, oxygen utilization, pathogenic potential, oxidative stress tolerance, and Gram-stain of the bacteria in the samples.

\section{Flagellin Concentration}

Initial data processing was executed in Excel 2016. First, a four-parametric logistic model was fitted to the standard curve using the 4PL-Curve Calculator from aatbio.com (AAT Bioquest., 2019). Given our observation that the emulsifiers decreased HEK cell activity, flagellin concentrations were normalized by use of absorbance values obtained from the resazurin assays; flagellin concentrations were divided by the ratio of the absorbance values from the samples over the average absorbance values for the standard curve of the same plate. Graphs were created using ggplot2. Since donors were observed separately and no replicate experiments per donor were performed, no statistical tests were performed for the flagellin data.

\section{Donor Diversity Analysis}

Next to the clustering of the donors in the dbRDA described above, we sought to assess the degree of susceptibility of the 10 donors to the effects of the emulsifiers, in an attempt to identify overall more or less susceptible individuals. Since literature describes no workflow for this purpose, we elaborated our own. Donors were ranked in terms of their susceptibility to the emulsifiers using several parameters: the $48 \mathrm{~h}$ production of the three most abundant SCFAs (acetate, propionate, and butyrate), the intact cell counts at T2, and the relative and absolute abundance of the most abundant OTU in the OTU-table, Escherichia/Shigella, at T2. These calculations were performed in Excel 2016.

First, to correct for the batch-effect, the control-values were subtracted from the treatment-values for each donor. Next, the corrected treatment values were summated for each donor, to obtain a single value that expressed the donor's susceptibility, and these values were then used to rank the donors from least to most susceptible for every parameter, visualized in bar graphs. This workflow was followed for each parameter.

\section{Comparison of Equivalent Emulsifier Concentrations}

Due to their stronger emulsifying properties, rhamnolipids and sophorolipids could reportedly be used in lower concentrations in food products than conventional chemical emulsifiers (Nitschke and Silva, 2018). Therefore, we sought to compare the effects of the chemical emulsifiers, CMC and P80, versus those of the biosurfactants, rhamnolipids, and sophorolipids, with regard to their impacts on the gut microbiota at equivalent emulsifying concentrations. Wilcoxon Rank Sum test was executed in $\mathrm{R}$ using the compare_means function on the same parameters we used to evaluate donor diversity (see Donor Diversity Analysis section). As equivalent concentrations we considered 
a $10 \times$ lower concentration of biosurfactants compared to the chemical emulsifiers, given that this is what industry reports (Van Haesendonck and Vanzeveren, 2006). Hence, we compared the condition of $0.5 \%(\mathrm{~m} / \mathrm{v})$ of chemical emulsifiers with that of $0.05 \%(\mathrm{~m} / \mathrm{v})$ of biosurfactancs and $0.05 \%(\mathrm{~m} / \mathrm{v})$ of chemical emulsifiers with $0.005 \%(\mathrm{~m} / \mathrm{v})$ of biosurfactants.

\section{RESULTS}

\section{Community Structure} Intact/Damaged Cell Counts

Analysis of intact and damaged cell populations with flow cytometry (SGPI-staining defines damage at the level of the cell membrane; Falcioni et al., 2008; Wlodkovic et al., 2009; Buysschaert et al., 2018), was used as a proxy for emulsifier toxicity. First, total and intact cell counts in the controls dropped by $14 \pm 2$ and $21 \pm 3 \%$, respectively, after $48 \mathrm{~h}$ in vitro incubation due to exhaustion of nutritional medium (Tables S1, S2 in Supplementary Data Sheet 1). When considering the impact of the emulsifiers, we observed that higher concentrations of rhamnolipids, sophorolipids, and soy lecithin resulted in significantly lower total and intact cell counts (Figures 1, 2 and Table S3 in Supplementary Data Sheet 1). At 0.5\% (m/v) rhamnolipids, intact cells decreased by $91 \pm 0 \%$ after $48 \mathrm{~h}$ compared to the control sample of T0. At $0.5 \%(\mathrm{~m} / \mathrm{v})$ sophorolipids, about $89 \pm 1 \%$, was lost and at $0.5 \%(\mathrm{~m} / \mathrm{v})$ soy lecithin, $87 \pm 1 \%$ was lost. The toxic effects were immediate for the sophorolipids and rhamnolipids, while for soy lecithin this decreasing effect only became significantly apparent after $24 \mathrm{~h}$ (T1; Figure 2). The impact of CMC and P80 toward the cell population was less pronounced. CMC even increased the total cell counts (not significantly) at higher concentrations, although the fraction of living cells remained unaffected for all CMC-conditions.

\section{Microbial Community}

The impact from the emulsifiers toward microbial community structure was assessed with 16S rRNA gene amplicon sequencing. First, the in vitro conditions had an impact on microbiota composition. While each donor showed a unique profile of microbial genera at the start of the experiment, more similar microbial community profiles were obtained upon incubation, primarily due to an increase in Escherichia/Shigella abundance from $0.02 \pm 0.02$ to $16 \pm 25 \%$, an increase in Bacteroides from $21 \pm 17$ to $42 \pm 20 \%$, a decrease in Faecalibacterium from $21 \pm 16$ to $1 \pm 1 \%$ and a decrease in Prevotella from $13 \pm 23$ to $2 \pm 4 \%$ (Figures S3-S5 in Supplementary Data Sheet 1). These alterations can be attributed to the feed composition, in which the relative enrichment in protein as well as the low carbohydrate concentration may have favored the growth of protein loving

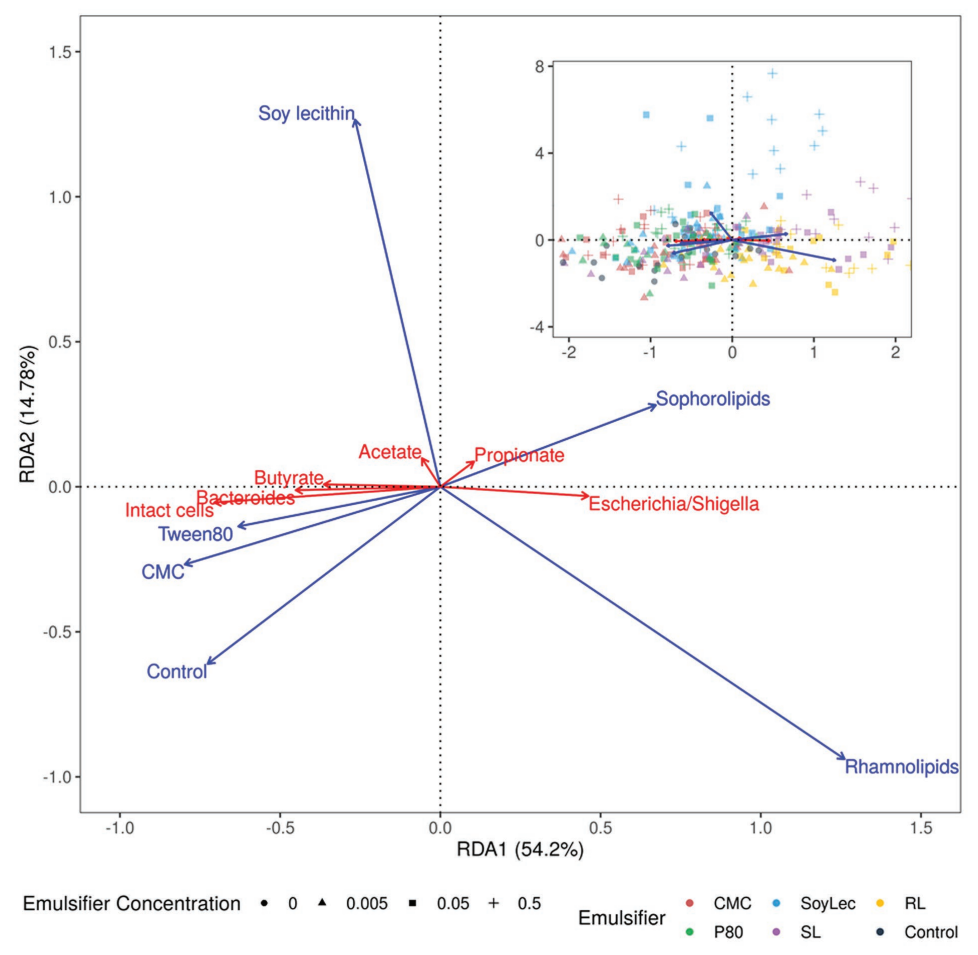

FIGURE 1 | Type II scaling triplot obtained using partial redundancy analysis of the microbial community detected after $48 \mathrm{~h}$ of in vitro batch incubations of fecal material from 10 human donors in sugar depleted medium supplemented with five emulsifiers at four concentrations. In the main figure, the intact cell count, the short chain fatty acid (SCFA)-levels, and the relative abundance of the top two genera are shown as response variables (red arrows), and emulsifier concentration are given as explanatory variables (blue arrows). The top right figure also displays the samples as sites. Axes are annotated with their contribution to the total variance. 
Escherichia/Shigella and versatile Bacteroides species over the more specialized and carbohydrate-loving Prevotella and Faecalibaterium species (Mu et al., 2016; Yao et al., 2016; Diether and Willing, 2019; Verhoog et al., 2019).
Independent from the in vitro effects, clear differences were noted between emulsifier treatments and controls, which were both emulsifier- and donor-dependent (Figure 3 and Figures S4, S5 in Supplementary Data Sheet 1). Where the
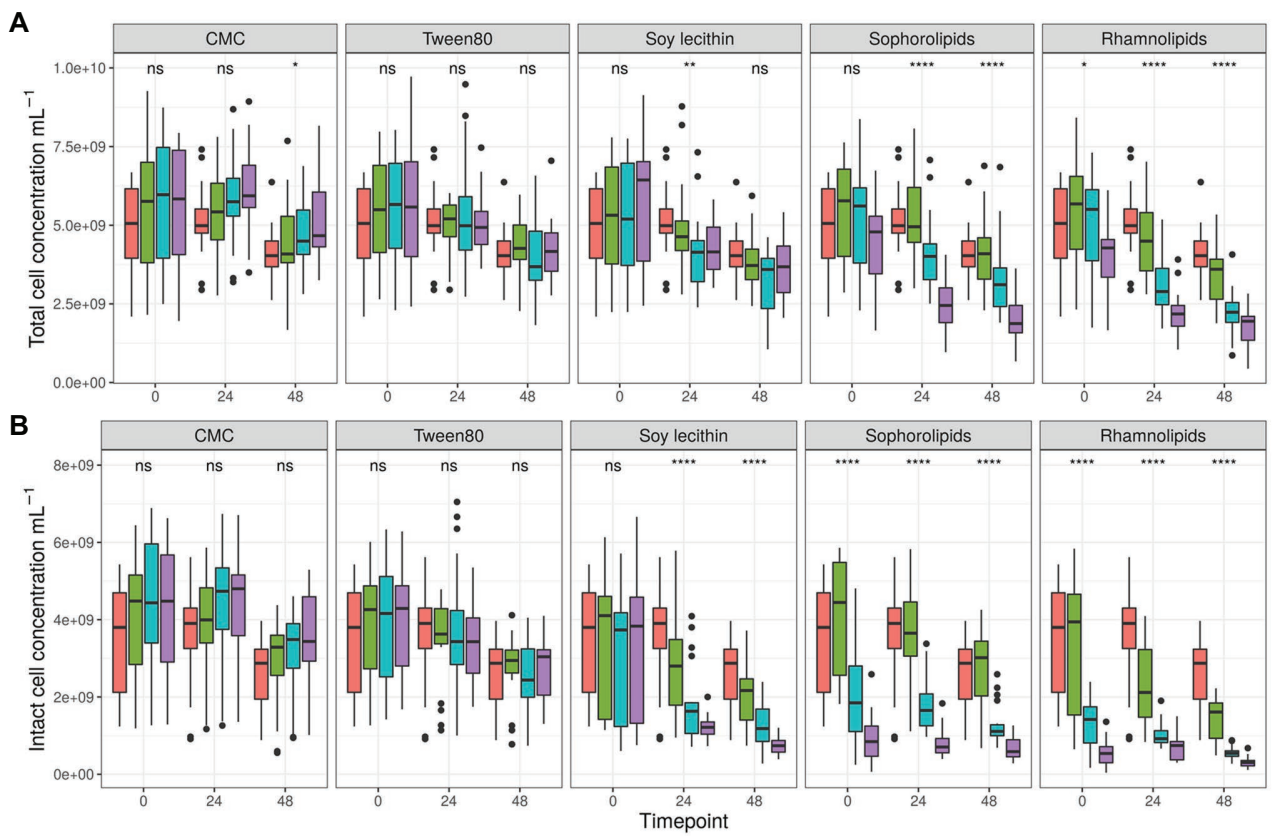

EM-conc. (\%) 追 0 白 0.005 追 0.05 追 0.5

FIGURE 2 | Average total (A) and intact (B) bacterial cell counts (cells/ml) detected during in vitro batch incubations of fecal material from 10 donors with sugar depleted medium supplemented with five emulsifiers at four concentrations. Samples were taken upon incubation (T0; $2-3 \mathrm{~h}$ after inoculation) as well as after $24 \mathrm{~h}$ (T1) and $48 \mathrm{~h}$ (T2) of incubation. Asterisks indicate significant differences detected with a Kruskal-Wallis test $(\alpha=0.05)$.
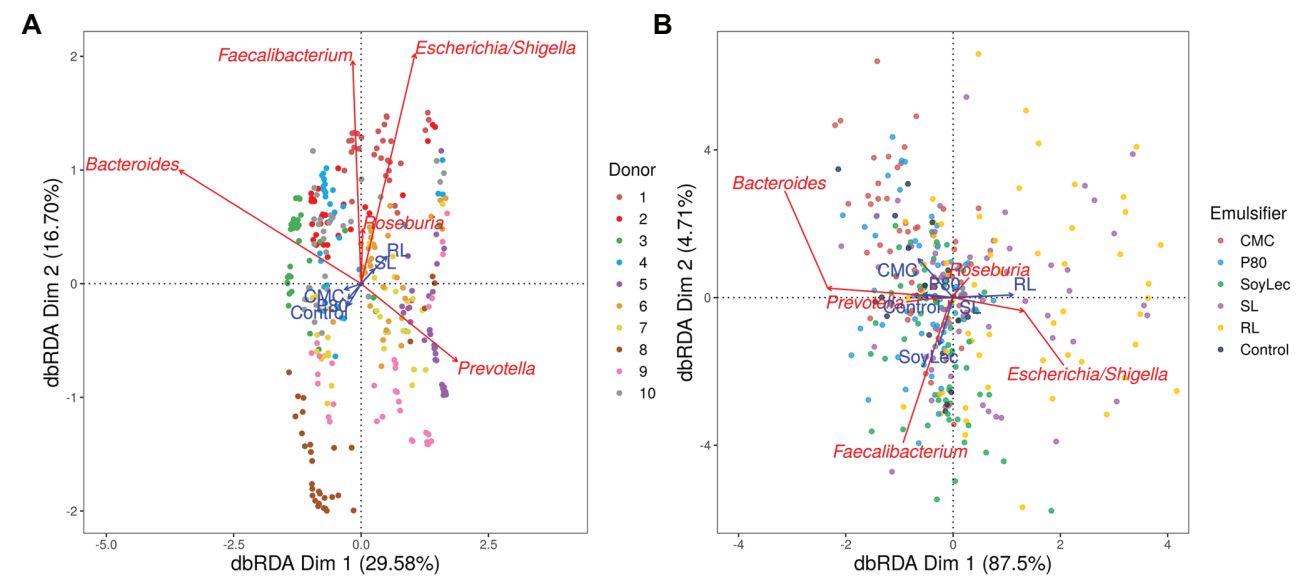

FIGURE 3 | Type I| scaling triplots obtained using partial distance based redundancy analysis of the microbial community composition detected using 16S rRNA gene amplicon sequencing after $48 \mathrm{~h}$ of in vitro batch incubations of fecal material from 10 donors with sugar depleted medium supplemented with five emulsifiers at four concentrations. Samples were taken upon incubation (TO; 2-3 h after inoculation) as well as after $24 \mathrm{~h}$ (T1) and $48 \mathrm{~h}$ (T2) of incubation. Factors donor, emulsifier, and emulsifier concentration were set as explanatory variables (blue arrows) and absolute abundances of genera as response variables (red arrows). Only the top five genera were displayed for adequate visibility. Axes are annotated with their contribution to the total variance. (A) The factor timepoint was partialled out. (B) The factors donor and timepoint were partialled out. 
effects of rhamnolipids and sophorolipids were most outspoken, the impact of soy lecithin was intermediary, and CMC and P80 had the smallest impacts (Figure 1). This was evidenced by significant drops in diversity indices upon incubation with rhamnolipids, sophorolipids and to a lesser extent soy lecithin (Figure 4). DESeq-analysis further revealed significant differential relative abundance of 36 genera, from which 23 were increased and 13 were suppressed, compared to the control condition (Figures 5, 6). Rhamnolipids triggered the strongest changes, with the three most increased genera being unclassified Enterobacteriaceae (L2FC = 3.85; padj < 0.0001), Fusobacterium (L2FC $=2.75 ;$ padj $<0.0001$ ), and Escherichia/Shigella (L2FC $=2.49 ;$ padj $<0.0001$ ) and the three most suppressed ones being unclassified Bacteroidetes (L2FC = -2.19; padj = <6,323E-4), Barnesiella $(\mathrm{L} 2 \mathrm{FC}=-2.09$; padj < 0.009), and Bacteroides (L2FC $=-2.02$; padj < 0.002). The top three most increased genera for sophorolipids were Escherichia/Shigella $(\mathrm{L} 2 \mathrm{FC}=1.86 ;$ padj $<0.043)$,
Acidaminococcus (L2FC $=1.80 ;$ padj $<0.0001)$, and Phascolarctobacterium $(\mathrm{L} 2 \mathrm{FC}=1.68$; padj $=<0.0001)$ and the three most decreased were unclassified Bacteroidetes (L2FC = -1.97; padj < 0.0001), Barnesiella (L2FC = -1.70; padj $=<0.0001$ ), and Bacteroides (L2FC $=-1.53$; padj $=3.034 \mathrm{E}$ $06)$. The top three most increased genera by soy lecithin were Acidaminococcus $(\mathrm{L} 2 \mathrm{FC}=1.23 ;$ padj $=0.016)$, Porphyromonadaceae_unclassified $(\mathrm{L} 2 \mathrm{FC}=1.19 ;$ padj $=0.017)$, and Sutterella $(\mathrm{L} 2 \mathrm{FC}=1.19$; padj $=0.004)$. Two significantly decreased genera were Flavonifractor (L2FC = -1.04; padj $=0.009)$ and Pseudoflavonifractor (L2FC $=-0.95$; padj $=0.015$; Figures 5, 6).

\section{Functional Analysis \\ Short Chain Fatty Acid}

Short chain fatty acids were analyzed to study how exposure to dietary emulsifiers affects the general microbial metabolic activity. We observed that SCFA-production was significantly
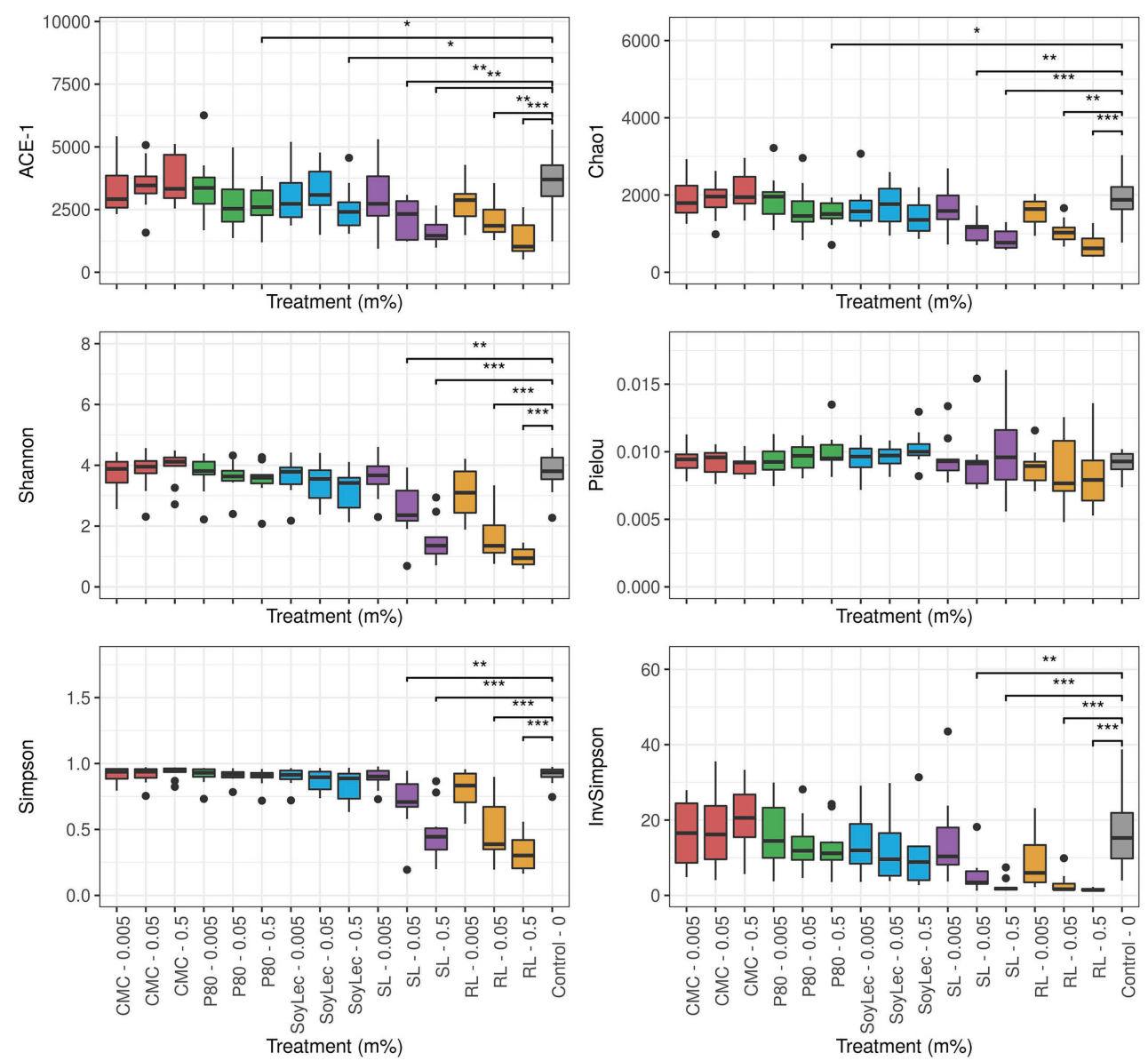

EM 追 CMC 自 SoyLec 甶 RL

FIGURE 4 | Diversity parameters of gut microbial community obtained after $48 \mathrm{~h}$ of in vitro batch incubations of fecal material from 10 donors with sugar depleted medium supplemented with five emulsifiers at four concentrations. Samples were taken upon incubation (T0; $2-3 \mathrm{~h}$ after inoculation) as well as after $24 \mathrm{~h}$ ( $\mathrm{T} 1$ ) and $48 \mathrm{~h}$ (T2) of incubation. Asterisks represent significant differences with control based on Wilcoxon Rank Sum tests with Holm's correction ( $\alpha=0.05)$. 


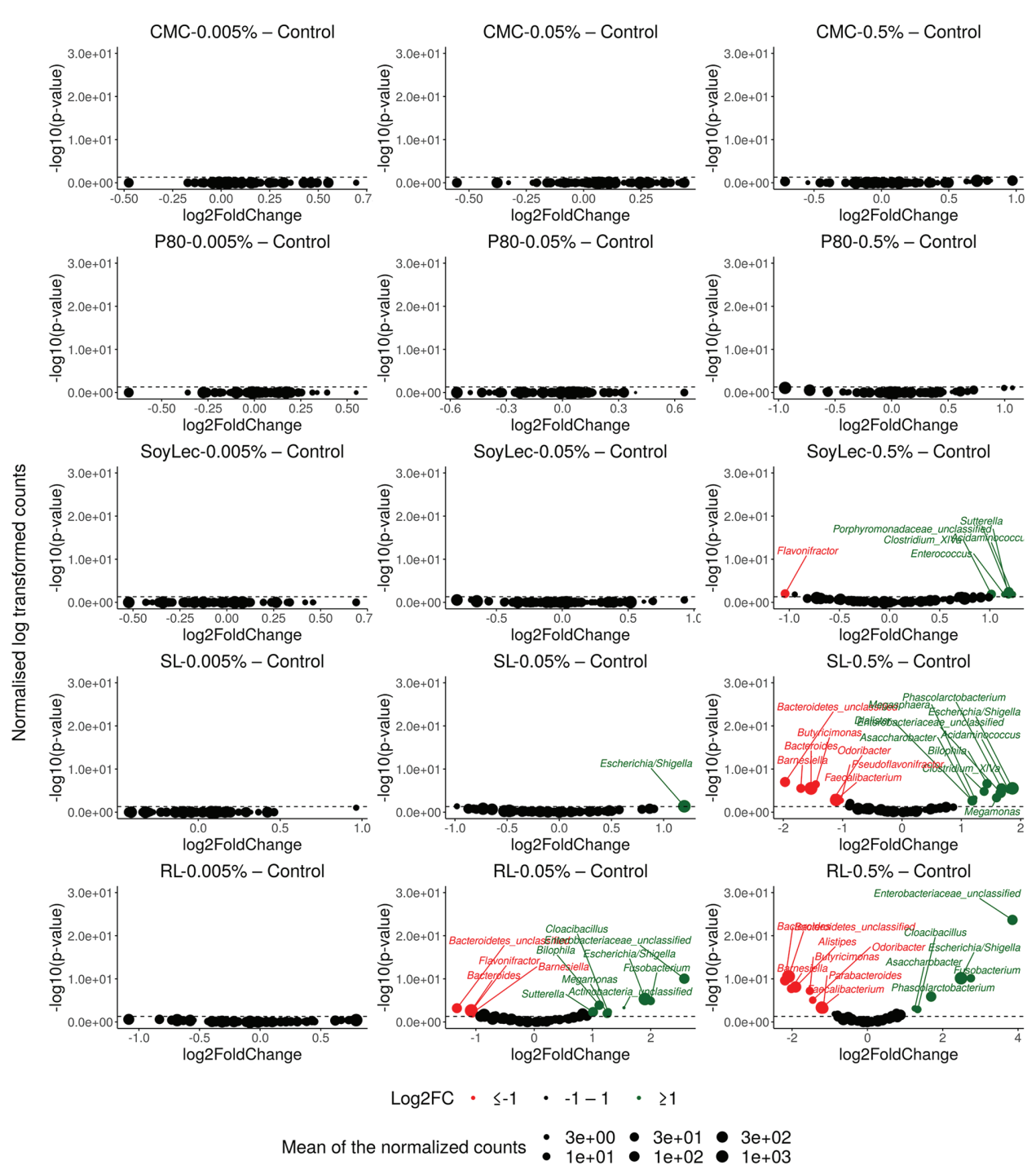

FIGURE 5 | Volcano plots indicating gut microbial community alterations after $48 \mathrm{~h}$ of in vitro batch incubations of fecal material from 10 donors with sugar depleted medium supplemented with five emulsifiers at four concentrations. Samples were taken upon incubation (T0; $2-3 \mathrm{~h}$ after inoculation) as well as after $24 \mathrm{~h}$ (T1) and $48 \mathrm{~h}$ (T2) of incubation. Log2FoldChange (L2FC) of genus abundances for all emulsifier treatments vs. the control are presented on the $x$-axis and the log transformed adjusted $p$-value is presented on the $y$-axis. Significantly increased or decreased genera are indicated in respectively green and red. The dashed line represents the significance threshold of $\alpha=0.05$.

and differently affected by rhamnolipids, sophorolipids, and soy lecithin, while no changes were observed for P80 and CMC (Figures 1, 7). The strongest impacts were noted for rhamnolipids, which, at $0.5 \%(\mathrm{~m} / \mathrm{v})$, significantly decreased total SCFA production by about $36 \pm 5 \%\left(p_{\text {Wilcox }}<0.0001\right)$ compared to the control condition. This decrease was mainly attributed to a $32 \pm 7 \%$ decrease in acetate production $\left(p_{\text {Wilcox }}<0.0001\right)$ compared to the control. Rhamnolipids at $0.5 \%(\mathrm{~m} / \mathrm{v})$ also reduced butyrate production by $96 \pm 6 \%$ compared to the control condition ( $\left.p_{\text {Wilcox }}<0.0001\right)$ while propionate production remained unaffected. Interestingly, incubation with $0.5 \%(\mathrm{~m} / \mathrm{v})$ sophorolipids also resulted in a decrease in butyrate production by $73 \pm 24 \%$ compared to the control $\left(p_{\text {Wilcox }}<0.0001\right)$, while propionate production increased by $88 \pm 50 \%\left(p_{\text {Wilcox }}=2.1 \mathrm{e}-04\right)$. Soy lecithin at $0.5 \%$ $(\mathrm{m} / \mathrm{v})$ significantly increased propionate production by $29 \pm 18 \%$ on average $\left(p_{\text {Wilcox }}=0.0089\right)$ and decreased butyrate production non-significantly by $34 \pm 25 \%$ on average $\left(p_{\text {Wilcox }}=0.035\right)$. No profound shifts in microbial fermentation activity were observed for incubations with CMC and P80.

\section{Metagenomic Prediction}

Other emulsifier related functional shifts were explored via metagenomic prediction using PICRUSt. These analyses predicted suppressing effects of rhamnolipids, sophorolipids on the pathways "Biosynthesis of other secondary metabolites," 


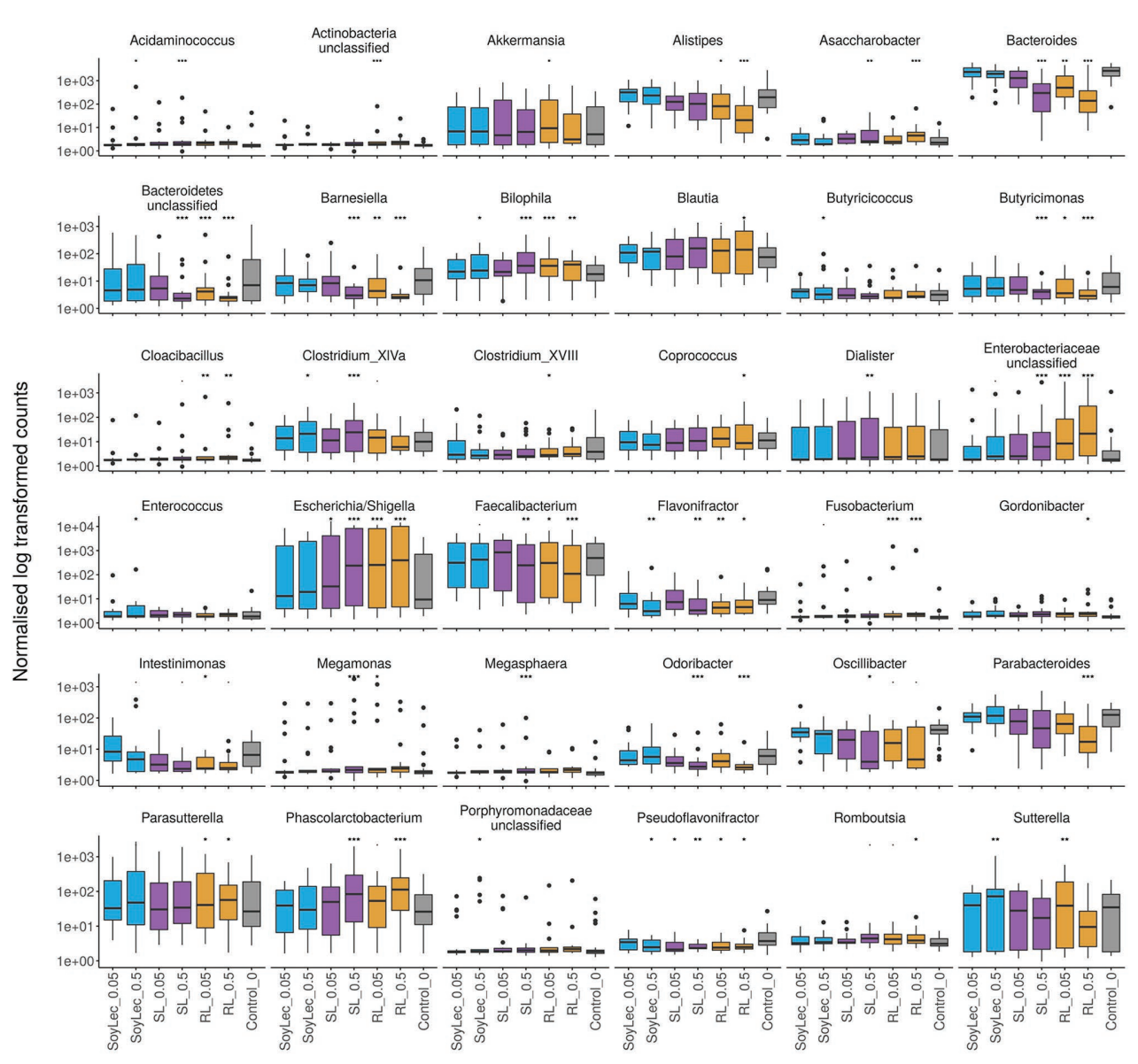

FIGURE 6 | Copy number corrected counts of significantly increased or decreased genera, obtained from DESeq analysis in R (version 3.4.2), after $48 \mathrm{~h}$ of exposure of gut microbial communities from 10 donors to soy lecithin, sophorolipids, and rhamnolipids during in vitro batch incubations. Asterisks represent significant differences with the control based on Wald tests $(\alpha=0.05)$.

"Cell growth and death," and "Signalling molecules and interaction" (Figure S6 in Supplementary Data Sheet 1). Possible significantly upregulated level 2 pathways were "Cell motility," "Cellular Processes and signaling," "Genetic information processing," "Lipid metabolism," "Membrane transport," "Metabolism," "Signal transduction," "Transcription," and "Xenobiotics degradation."

Plugging the data into the Bugbase-webtool revealed a significantly stimulating effect of sophorolipids and rhamnolipids on the formation of biofilms and mobile elements, stress tolerance and increased abundance of potential pathogens, Gram negative and facultative anaerobic bacteria all properties related to the Proteobacteria phylum. This coincides with our observation of an increased abundance of Escherichia/Shigella (Supplementary Data Sheet 2).

\section{Flagellin Levels}

In order to validate the PICRUSt prediction of a higher motility potential, a HEK-blue mTLR5 reporter cell assay was used for the detection of bacterial flagellin. The response of the flagellin concentrations to the emulsifiers was found to be largely donor-dependent, and inconsistent shifts were observed in function of incubation time (Figure 8). Shifts in flagellin levels upon emulsifier dosage were variable, meaning that the prediction of higher motility by PICRUSt could not be substantiated.

\section{Donor Diversity}

For all endpoints, inter-individual variability was observed in response to the in vitro incubations and emulsifier treatments (Figures S4, S5, S7 in Supplementary Data Sheet 1). In terms of community structure, Figure 3A shows that each donor clusters separately. This clustering was found to be significant $\left(p_{\mathrm{dbRDA}}<0.05\right)$.

To assess in more detail whether there was coherence in the read-outs with respect to donor susceptibility, we ranked the donors according to their response on the most relevant parameters, i.e., intact cell counts, production of the most important SCFA (acetate, propionate, and butyrate), and the absolute and relative abundance of Escherichia/Shigella.

We observed that the susceptibility of the donors to the effects of the emulsifiers depended on the targeted parameter (Figure 9). Some donors consistently ranked as highly 


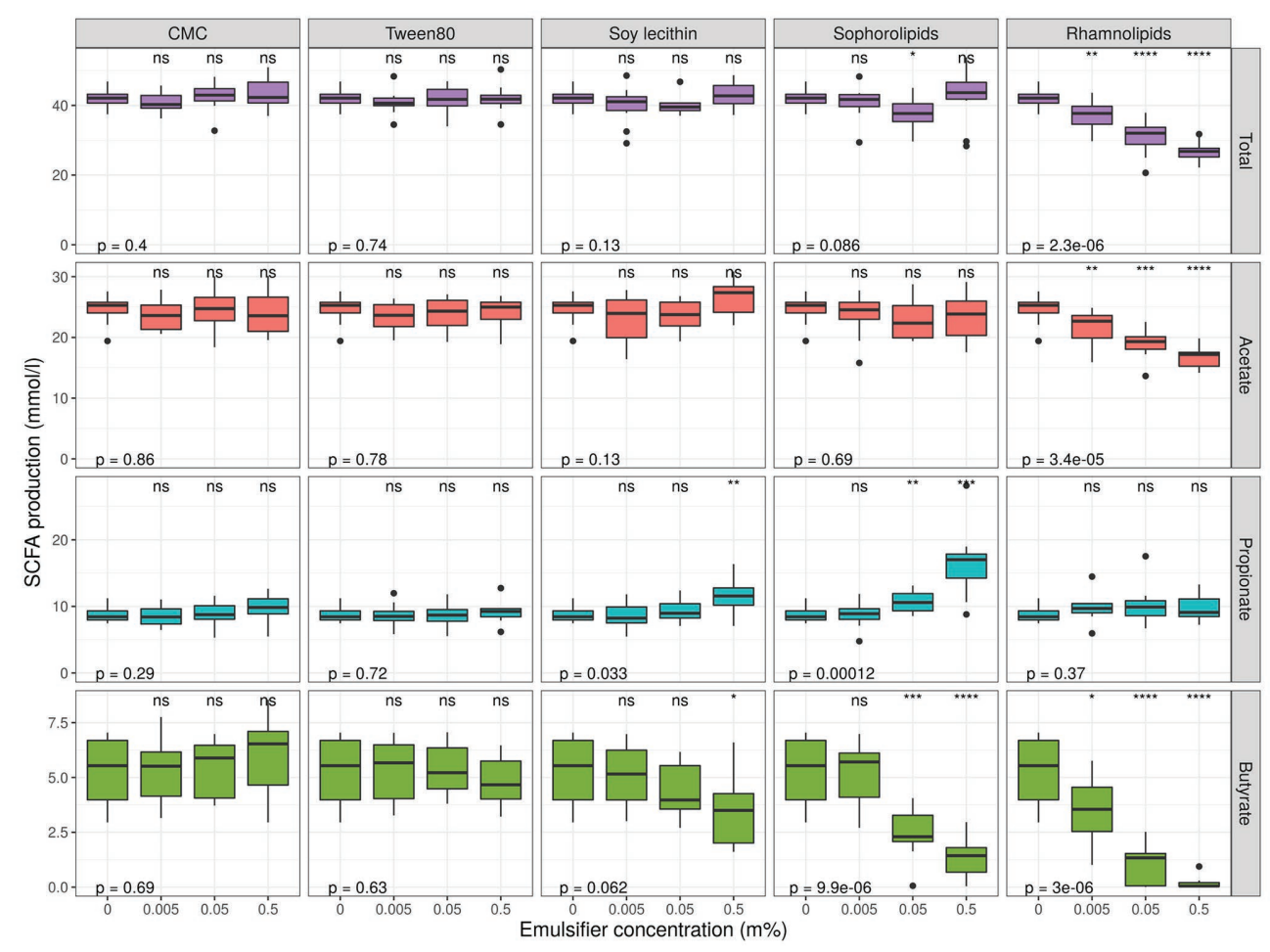

FIGURE 7 | Short chain fatty acid production levels over $48 \mathrm{~h}$ of incubation of fecal material from 10 donors in sugar depleted medium supplemented with five emulsifiers at four concentrations. Samples were taken upon incubation (TO; 2-3 h after inoculation) as well as after $24 \mathrm{~h}$ (T1) and $48 \mathrm{~h}$ (T2) of incubation. Asterisks indicate significant differences with the control [0\% (m/v)], calculated with Wilcoxon Rank Sum tests with Holm's correction. $p$-values indicate results of general Kruskal-Wallis tests $(\alpha=0.05)$.

susceptible (D9) or less susceptible (D6 and D1), while for other donors, ranking was more variable over the different parameters.

\section{Equivalent Emulsifier Concentrations}

We sought to compare the effects of biosurfactants versus those of conventional chemical emulsifiers. When comparing $0.5 \%$ $(\mathrm{m} / \mathrm{v})$ of chemical emulsifier with $0.05 \%(\mathrm{~m} / \mathrm{v})$ of biosurfactant - the concentrations most representative of the currently applied levels of dietary emulsifiers - the previously described effects of the biosurfactants (decreased levels of acetate, butyrate, intact and total cell concentration, and increased abundance of propionate and increased abundance of Escherichia/Shigella) were significant compared to the effects of the chemical emulsifiers (Table S4 in Supplementary Data Sheet 1). When comparing $0.05 \%(\mathrm{~m} / \mathrm{v})$ of chemical emulsifier with $0.005 \%(\mathrm{~m} / \mathrm{v})$ of biosurfactant, the effects were not significantly different, except for the effects of rhamnolipids on the cell count data.

\section{DISCUSSION}

We found dietary emulsifiers to significantly alter human gut microbiota toward a composition and functionality with potentially higher pro-inflammatory properties. While donor-dependent differences in microbiota response were observed, our in vitro experimental setup showed these effects to be primarily emulsifierdependent. Rhamnolipids and sophorolipids had the strongest impact with a sharp decrease in intact cell counts, an increased abundance in potentially pathogenic genera-like Escherichia/Shigella and Fusobacterium, a decreased abundance of beneficial Bacteroidetes and Barnesiella, and a predicted increase in flagellar assembly and general motility. The latter was not substantiated through direct measurements, though. The effects were less pronounced for soy lecithin, while chemical emulsifiers P80 and CMC showed the smallest effects. Short chain fatty acid production, with butyrate production, in particular, was also affected by the respective emulsifiers, again in an emulsifier- and donordependent manner.

One of the most profound impacts of emulsifier treatment toward gut microbiota was the decline in intact microbial cell counts. The degree of microbiome elimination in this study seems comparable to what has been observed for antibiotic treatments (Francino, 2016; Guirro et al., 2019). Since antibiotics are considered detrimental for gut ecology, this may serve as a warning sign with respect to emulsifier usage. Emulsifiers also act as surfactants, which are known for their membrane solubilizing properties (Jones, 1999). The fact that the observed decline in microbial viability was dependent on emulsifier dose and on the emulsifying potential 


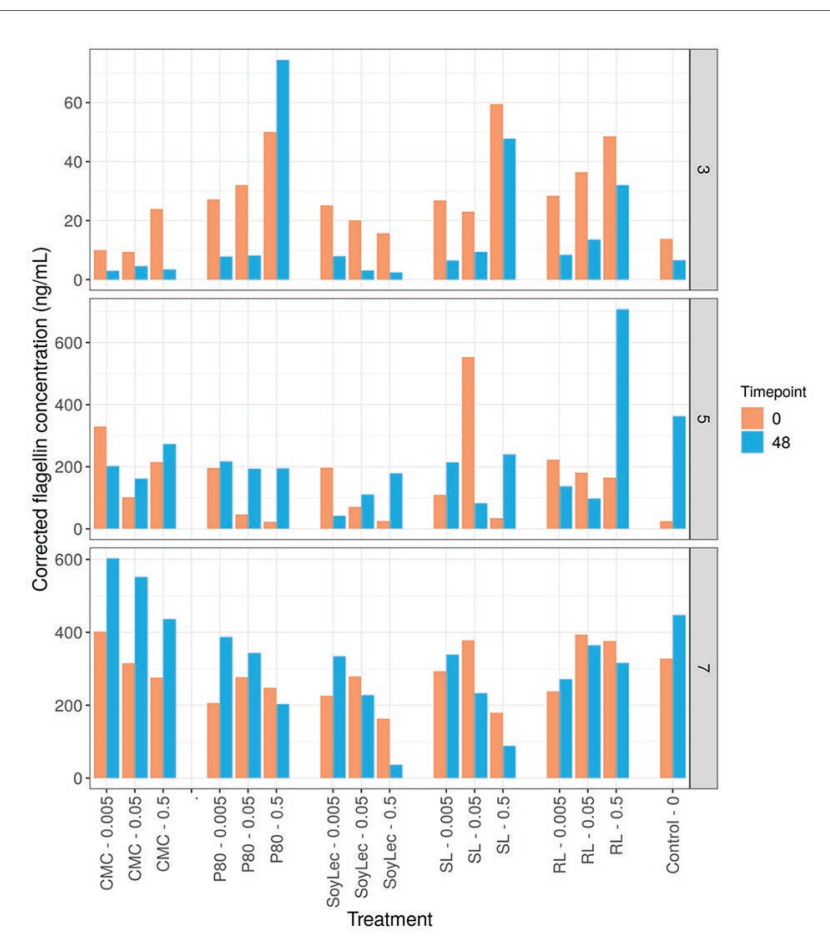

FIGURE 8 | Flagellin concentrations obtained using mTLR5 HEK blue reporter cells for three donors at the start (TO) and the end (T2) of in vitro $48 \mathrm{~h}$ batch experiments of fecal material from 10 donors in sugar depleted medium supplemented with five emulsifiers at four concentrations. Donors for the flagellin assay were selected based on their low (D3), high (D7), and intermediate (D5) metabolic responses to the emulsifiers.

of the supplemented compound, as measured by the aqueous surface tension reduction (Table 1), leads us to conclude that the dietary emulsifiers attack the bacterial cells principally at the level of the cell membrane.

We also found this antimicrobial effect from the tested emulsifiers to be highly selective, confirming previous observations by Moore (1997) who showed that the effects of surfactants are dependent on the bacterial species. We found the Escherichia/Shigella genus to be particularly resistant against the surfactants antimicrobial effects. This agrees with Kramer et al. (1984) and Nickerson and Aspedon (1992) who demonstrated the surfactant resistance of enteric bacteria Enterobacter cloacae and E. coli against sodium dodecyl sulfate. They showed that this resistance is widespread among Enterobacteriaceae, that it is energy-dependent, and that exposure to sodium dodecyl sulfate altered the expressions of 19 proteins, of which three were later tentatively identified as clpP, clpB, and clpX intracellular proteases (Rajagopal et al., 2002). Also membrane-derived oligosaccharides, present in the periplasm of Gram negative bacteria, were found to be essential to the detergent-resistant properties of E. coli (Rajagopal et al., 2003). Even though this could explain the increased abundance in this study for Enterobacteriaceae and Escherichia/Shigella, the mechanism by which other species are (un)affected by the emulsifiers is not known.
Interestingly, our prediction of altered functionality using PICRUSt analysis indicated a potential increase in levels of motility-genes, even though we could not confirm increased flagellin levels by direct measurement. Increased motility has been observed upon addition of emulsifiers before (Lock et al., 2018). This finding may again be related to the increased relative abundance of Escherichia/Shigella, since these are known flagella (gene)-bearers (Girón, 1995; Tominaga et al., 2001; Mittal et al., 2003). Increased flagellin levels constitute a potential health risk as flagellin is considered an important virulence factor; this particular microbe-associated molecular pattern may trigger inflammation upon binding to TLR5. A higher degree of flagellation would thus represent more motile bacteria and result in a gut microbiome that is able to more aggressively penetrate the mucus layer and subsequently reach gut epithelial cells (Ramos et al., 2004; Chassaing et al., 2015). Chassaing et al. $(2015,2017)$ demonstrated possible consequences for the host: an increased inflammatory response in both gut and body, contributing to increased adiposity and weight gain.

Effects toward microbial metabolic functionality, as measured by SCFA-levels, were emulsifier-dependent. Nevertheless, consistent shifts in SCFA-profiles were observed for all three emulsifiers for which significant alterations were visible, i.e., a decrease in butyrate and an increase in propionate production. For sophorolipids, the strong increase in propionate production can be related to the increased abundance of Phascolarctobacterium. This genus is known to produce propionate from succinate produced by E. coli cells (Del Dot et al., 1993). For rhamnolipids, we assume that similar crossfeeding interactions occurred, even though propionate levels remained stable. We hypothesized that an increased propionate production by the Escherichia/Phascolarctobacterium consortium is counteracted by the higher antimicrobial activities from the rhamnolipids at increasing concentrations. The fact that L-rhamnose is a well-known propionate precursor (Reichardt et al., 2014), and that rhamnolytic pathways have been observed in E. coli and other Gammaproteobacteria (Rodionova et al., 2013) further support this idea. The increase in acetate and propionate production observed with soy lecithin can be linked to the metabolism of glycerol (De Weirdt et al., 2010). This is in agreement with the upregulated abundances of the Enterococcus and Clostridium genera at higher levels of soy lecithin, since these genera are known to metabolize glycerol (Bradbeer, 1965; Bizzini et al., 2010) as well as choline (Martínez-del Campo et al., 2015). Also the increased abundance of the Acidaminoccus genus would correspond with the increased acetate production (Chang et al., 2010). These findings indicate that observed shifts in SCFA-production can be attributed to shifts in microbial composition.

Whether the altered SCFA-production and -levels are positive or negative for host health is ambiguous. On the one hand, decreased butyrate levels can be considered negative, since butyrate is known to protect the gut epithelium from inflammation and cancerous growth (Canani et al., 2011; Cani, 2017; Liu et al., 2018). On the other hand, propionate is also considered a health-promoting SCFA (Hosseini et al., 2011; Weitkunat et al., 2016; Louis and Flint, 2017). 


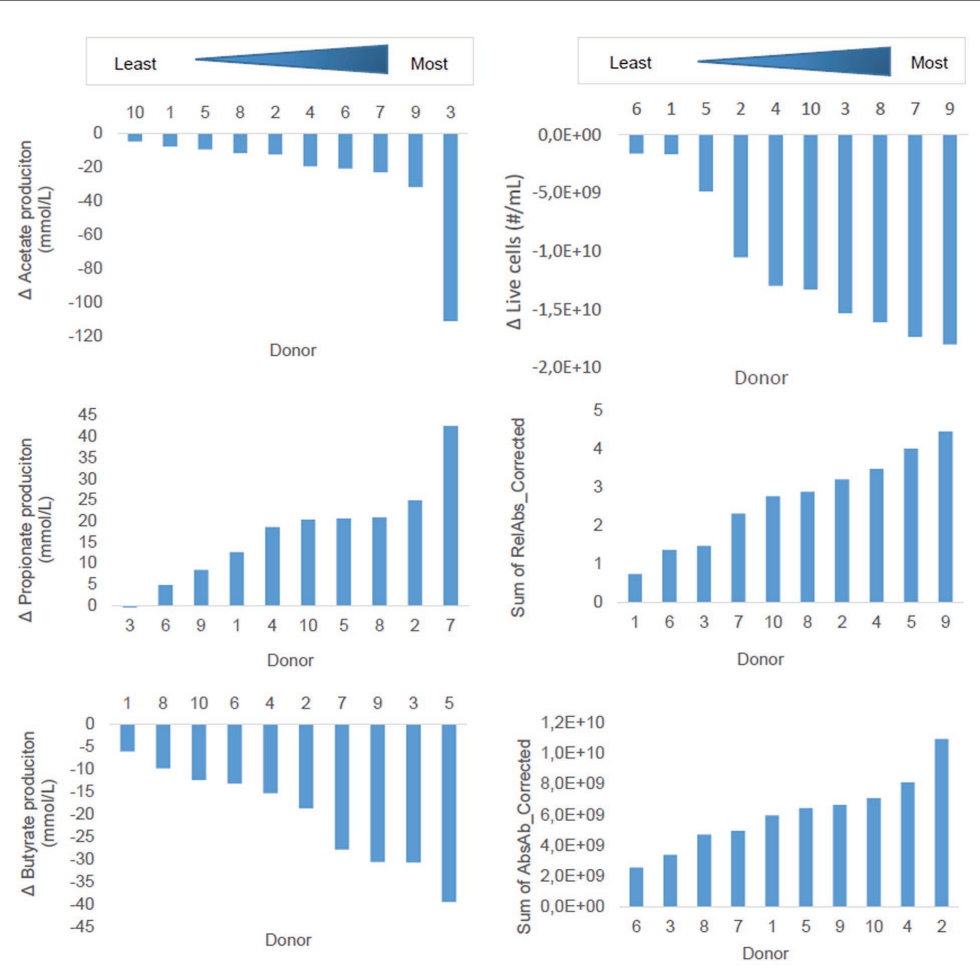

FIGURE 9 | Ranking of donors of fecal material for $48 \mathrm{~h}$ batch incubations in sugar depleted medium supplemented with five emulsifiers at four concentrations. Donors were ranked along the principal parameters impacted by the emulsifiers. Measures were calculated based on cumulative (sum of all treatments) change in $48 \mathrm{~h}$ production of SCFA or cumulative $48 \mathrm{~h}$ change in living cell counts, relative or absolute abundances of Escherichia/Shigella.

Propionate production is related to decreased lipogenesis in the liver and is supposed to enhance satiety mechanisms, which would lower the chance of developing obesity (Hosseini et al., 2011; Weitkunat et al., 2016). It is, however, questionable whether such benefit may predominate the purported negative effects from the observed antimicrobial effects and the increase in pro-inflammatory markers, such as flagellated microbiota and a drop in butyrate production.

Another point relating to health effects entails that rhamnolipids, sophorolipids, and - to a lesser extent - soy lecithin significantly decreased diversity parameters. Decreased microbiome diversity frequently occurs with compromised health conditions such as obesity, insulin resistance, dyslipidemia (Turnbaugh et al., 2009; Le Chatelier et al., 2013), Type 1 diabetes (Patterson et al., 2016), heart failure (Luedde et al., 2017), and inflammatory bowel disease (Arumugam et al., 2011; Chassaing et al., 2015). The increased prevalence of the Escherichia/Shigella genus has also been observed with multiple metabolic conditions (Lupp et al., 2007; Lecomte et al., 2015; Shin et al., 2015). Increased Enterobacteriaceae abundance was previously linked with an increased gut permeability (Pedersen et al., 2018), the consumption of high fat diets (Fei and Zhao, 2013; Lecomte et al., 2015; He et al., 2018), colitis (Lupp et al., 2007), cardiovascular disease (Jie et al., 2017), diabetes (Allin et al., 2015; Deschasaux et al., 2019), and even undernourishment and iron deficiency anemia (Shin et al., 2015;
Muleviciene et al., 2018). With respect to soy lecithin, metabolism of phosphatidylcholine by the gut microbiota has been linked to cardiovascular disease (Wang et al., 2011; Tang and Hazen, 2014). We also found an increased abundance of the genus Sutterella, a bacterium that has been linked to autism spectrum disorders (Wang et al., 2013) and gut inflammation, notably by IgA-degradation (Moon et al., 2015; Kaakoush, 2020). Overall, we can, thus, conclude that exposure of the gut microbiota to dietary emulsifiers may result in profound compositional shifts, previously related to adverse health outcomes.

An important consideration is whether the observed in vitro effects would also take place in an in vivo setting. This will depend on a number of diet- and host-related factors. First, we found that the observed effects were concentration- and emulsifier-dependent. Choosing an emulsifier-concentration combination that minimizes adverse microbial impacts, without compromising food technical properties, could thus be a strategy to mitigate the harmful effects of food emulsifiers. Second, emulsifier concentration will continuously alter during gastrointestinal digestion, but the impact of the dilution with accompanying food products, excretion of digestive fluids, or absorption of water from the gut lumen on the final concentration reaching the gut microbiota has so far not yet been studied. Third, digestion by human enzymes will alter chemical structure of the emulsifiers. While CMC is resistant to breakdown by human digestive enzymes (Joint FAO/WHO Expert Committee 
TABLE 1 | Emulsifier characteristics of sodium carboxymethylcellulose, Span60, P80, phosphatidylcholine (major component of soy lecithin), sophorolipids, and rhamnolipids.

\begin{tabular}{|c|c|c|c|c|c|}
\hline & MW (g/mol) & $\mathrm{CMC}^{\star}$ (mg/L) & Source & Min. S.T. ${ }^{\star \star}(\mathrm{mN} / \mathrm{m})$ & Source \\
\hline Carboxymethylcellulose & $250,000^{1,2}$ & - & & - & \\
\hline $\begin{array}{l}\text { Sorbitan tristearate } \\
\text { (Span60) }\end{array}$ & $430.62^{3}$ & 21.53 & Michor and Berg, 2015 & 62 & Parreidt et al., 2018 \\
\hline \multirow[t]{3}{*}{$\mathrm{P} 80$} & $1,310^{4}$ & $0.012 \mathrm{mM}$ & Pogorzelski et al., 2012 & $+/-43$ & Bąk and Podgórska, 2016 \\
\hline & & $\begin{array}{r}=15.72 \mathrm{mg} / \mathrm{L} \\
0.033 \mathrm{mM}\end{array}$ & $\begin{array}{l}\text { Bąk and Podgórska, } \\
2016\end{array}$ & $36-38$ & Szymczyk et al., 2018 \\
\hline & & $=43.23 \mathrm{mg} / \mathrm{L}$ & & & \\
\hline \multirow[t]{3}{*}{ Phophatidylcholine } & $758.1^{5}$ & 0.998 PC (16:0/16:0) & Zhang et al., 2017 & 38 mN/m (PC) & Wu and Wang, 2003 \\
\hline & $643.9 \mathrm{~g} / \mathrm{mol}^{6}$ & 0.001 PC (18:0/18:0) & Zhang et al., 2017 & (21 mN/m for $\mathrm{Pl})$ & Wu and Wang, 2003 \\
\hline & $786.1^{7}$ & 13,600 (lecithin) & Wu and Wang, 2003 & & \\
\hline \multirow[t]{5}{*}{ Sophorolipids } & $706^{8}$ & 150 & Kim et al., 2005 & 48 & Kim et al., 2005 \\
\hline & & $\begin{array}{l}27.17 \\
10\end{array}$ & $\begin{array}{l}\text { Daverey and Pakshirajan, } \\
2010\end{array}$ & $\begin{array}{l}34.18 \\
36\end{array}$ & $\begin{array}{l}\text { Daverey and Pakshirajan, } \\
2010\end{array}$ \\
\hline & & $20-130$ & Otto et al., 1999 & $31-34.2$ & Otto et al., 1999 \\
\hline & & & Ma et al., 2012 & $32.23-42.13$ & $\begin{array}{l}\text { Develter and Lauryssen, } \\
2010\end{array}$ \\
\hline & & & & & Ma et al., 2012 \\
\hline \multirow[t]{5}{*}{ Rhamnolipids } & $650.79^{9}$ & $50-500$ & Li et al., 2019 & 28 & Li et al., 2019 \\
\hline & & 18.75 & Moussa et al., 2014 & 34 & Moussa et al., 2014 \\
\hline & & $15-20$ & Hörmann et al., 2010 & $25-30$ & Van Bogaert, 2008 \\
\hline & & 30 & Vu et al., 2015 & 29.4 & Hörmann et al., 2010 \\
\hline & & & & $30-35$ & Vu et al., 2015 \\
\hline
\end{tabular}

${ }^{*}$ Critical micelle concentration.

**Minimal surface tension in aqueous solution.

${ }^{1}$ https://www.sigmaaldrich.com/catalog/product/aldrich/419311?lang=en\&region=CA (4-06-2020).

${ }^{2}$ https://pubchem.ncbi.nlm.nih.gov/compound/Carboxymethylcellulose-sodium (4-06-2020).

${ }^{3}$ https://www.sigmaaldrich.com/catalog/product/sial/85546?lang=en\&region=CA (4-06-2020).

${ }^{4}$ https://www.sigmaaldrich.com/content/dam/sigma-aldrich/docs/Sigma-Aldrich/Product_Information_Sheet/p8074pis.pdf (4-06-2020).

${ }^{5}$ https://pubchem.ncbi.n/m.nih.gov/compound/Soybean-lecithin (23-01-2020).

${ }^{6}$ https://pubchem.ncbi.nlm.nih.gov/compound/57369748 (23-01-2020).

7https://pubchem.ncbi.nlm.nih.gov/compound/6441487 (23-01-2020).

${ }^{8}$ https://pubchem.ncbi.nlm.nih.gov/compound/Sophorolipid (23-01-2020).

${ }^{9}$ https://pubchem.ncbi.nlm.nih.gov/compound/5458394 (23-01-2020).

on Food Additives, 1973), P80 and soy lecithin can be hydrolyzed by pancreatic lipases. For P80, only the polyethylene-sorbitan unit may reach the colon (Aguilar et al., 2015). Soy lecithin is mostly absorbed as lysolecithin and free fatty acids, but its detection in faces indicates that some fraction reaches the colon (Mortensen et al., 2017), where the choline and glycerol moieties are metabolized by the gut microbiota (Tang and Hazen, 2014). For both of these compounds, it will thus be necessary to verify if and to what extent the observed effects occur in vivo as well. With respect to rhamnolipids and sophorolipids, no literature is currently available on their digestion by human enzymes. Preliminary data from our side, however, have indicated only one alteration, a deacetylation of the sophorose units of the sophorolipids (Supplementary Data Sheet 3). These compounds may thus readily reach the colon and interact with the endogenous microbiota. Fourth, other dietary constituents in the gut, primarily lipids, and bile salts, will interact with emulsifiers (Naso et al., 2019). The mucus layer overlying the gut epithelium and the $\mathrm{pH}$-fluctuations throughout the gut are other elements that may affect emulsifier-microbiota interactions. Finally, the total levels of emulsifiers consumed by an individual will determine both acute and chronic effects.

A last important element in the putative health impact from dietary emulsifiers concern's interindividual variability. An individual's unique microbiota and metabolism are important determinants of the potential health effects dietary emulsifiers could cause. While the overall effects from the different emulsifiers toward microbiota composition and functionality were quite consistent in our study, important interindividual differences in susceptibility of the microbiota were noted. Understanding what underlying factors and determinants drive this interindividual variability will be crucial to future health risk assessment of novel and existing dietary emulsifiers.

Food additives have come under scrutiny with respect to their impact on human health. Additives like colorants, artificial sweeteners, nitrites $\left(\mathrm{NaNO}_{2}\right)$, and high fructose corn syrup have been associated with hyperactivity, cancer development, gastric cancer, and obesity, respectively (Arnold et al., 2012; Bryan et al., 2012; Payne et al., 2012; Carocho et al., 2014). 
In answer to these public concerns, a clean label movement has developed in the food industry that aims to provide food products with a more natural image. In this light, we investigated whether rhamnolipids and sophorolipids, two biotechnological emulsifiers, would yield less of an impact on the gut microbiota than the mainstream chemical emulsifiers, CMC and P80. Our results showed however, that rhamnolipids and sophorolipids, of all emulsifiers in this study, had the strongest impact on microbiota composition and functionality, even when equivalent concentrations were taken into account. Further analysis of our data showed that the observed effects toward the microbiota can potentially be linked to their higher emulsifying potential. All of this indicates that rhamnolipids and sophorolipids are probably no appropriate alternatives to conventional emulsifiers unless they are used at substantially lower concentrations. We note, however, that the model used in this study has impacted the microbiota as well. More research must thus point out whether the effects prevail in different models, as wel as in vivo and if so, whether concentrations can be kept low enough to avoid alleged adverse health effects.

\section{DATA AVAILABILITY STATEMENT}

The datasets presented in this study can be found in online repositories. The names of the repository/repositories and accession number(s) can be found in the article/ Supplementary Material.

\section{ETHICS STATEMENT}

The studies involving human participants were reviewed and approved by Ghent University Hospital (registration number BE670201836318). Written informed consent for participation was not required for this study in accordance with the national legislation and the institutional requirements.

\section{REFERENCES}

AAT Bioquest (2019). Quest Graph ${ }^{\text {TM }}$ Four Parameter Logistic (4PL) Curve Calculator. Available at: https://www.aatbio.com/tools/four-parameter-logistic4pl-curve-regression-online-calculator (Accessed March 13, 2020).

Adams, W., Bas, H., Boutte, T., Bueschelberger, H. -G., Cooper, J. M., Cotrell, T., et al. (2004). "Emulsifiers in food technology" in Emulsifiers in food technology. ed. R. J. Whitehurst (John Wiley and Sons Ltd.), 104.

Aguilar, F., Crebelli, R., di Domenico, A., Dusemund, B., Frutos, M. J., Galtier, P., et al. (2015). Scientific Opinion on the re-evaluation of polyoxyethylene sorbitan monolaurate (E 432), polyoxyethylene sorbitan monooleate (E 433), polyoxyethylene sorbitan monopalmitate (E 434), polyoxyethylene sorbitan monostearate (E 435) and polyoxyethylene sorbita. EFSA J. 13:4152. doi: 10.2903/j.efsa.2015.4152

Allin, K. H., Nielsen, T., and Pedersen, O. (2015). Mechanisms in endocrinology: gut microbiota in patients with type 2 diabetes mellitus. Eur. J. Endocrinol. 172, R167-R177. doi: 10.1530/EJE-14-0874

Anderson, J. R., Carroll, I., Azcarate-Peril, M. A., Rochette, A. D., Heinberg, L. J., Peat, C., et al. (2017). A preliminary examination of gut microbiota, sleep, and cognitive flexibility in healthy older adults. Sleep Med. 38, 104-107. doi: 10.1016/j.sleep.2017.07.018

\section{AUTHOR CONTRIBUTIONS}

This research was performed under promotorship of TW, JC, and AR. LM was responsible for the execution of all experiments, data visualization, and statistical analysis, as well as the assembly and submission of the manuscript. CC assisted in the execution of the PICRUSt algorithm. DESeq analysis was performed in collaboration with KP. Library preparation and 16S rRNA gene amplicon sequencing was performed by LR, our collaborator within JR group. All authors contributed to the article and approved the submitted version.

\section{FUNDING}

This study was funded by a scholarship from the UGent special research fund (BOF17/DOC/312-file number: 01D31217), a UGent research grant (BOF17-GOA-032) and the FWO-EOS grant MiQuant.

\section{ACKNOWLEDGMENTS}

We thank prof. Inge Van Bogaert (Inbio, Ghent University) for providing us with sophorolipids samples. We also thank Jo Devrieze for providing feedback to our manuscript before submission.

A preprinted version of this manuscript is available at biorXiv: https://biorxiv.org/cgi/content/short/2020.06.28.174946v1.

\section{SUPPLEMENTARY MATERIAL}

The Supplementary Material for this article can be found online at: https://www.frontiersin.org/articles/10.3389/fmicb.2020.577474/ full\#supplementary-material

Arnold, L. E., Lofthouse, N., and Hurt, E. (2012). Artificial food colors and attention-deficit/hyperactivity symptoms: conclusions to dye for. Neurotherapeutics 9, 599-609. doi: 10.1007/s13311-012-0133-x

Arumugam, M., Raes, J., Pelletier, E., Le Paslier, D., Yamada, T., Mende, D., et al. (2011). Enterotypes of the human gut microbiome. Nature 473, 174-180. doi: 10.1038/nature09944

Asioli, D., Aschemann-Witzel, J., Caputo, V., Vecchio, R., Annunziata, A., Næs, T., et al. (2017). Making sense of the "clean label" trends: a review of consumer food choice behavior and discussion of industry implications. Food Res. Int. 99, 58-71. doi: 10.1016/j.foodres.2017.07.022

Bąk, A., and Podgórska, W. (2016). Interfacial and surface tensions of toluene/ water and air/water systems with nonionic surfactants Tween 20 and Tween 80. Colloids Surf. A Physicochem. Eng. Asp. 504, 414-425. doi: 10.1016/j. colsurfa.2016.05.091

Bischoff, S. C. (2011). "Gut health": a new objective in medicine? BMC Med. 9:24. doi: 10.1186/1741-7015-9-24

Biswal, D. R., and Singh, R. P. (2004). Characterisation of carboxymethyl cellulose and polyacrylamide graft copolymer. Carbohydr. Polym. 57, 379-387. doi: 10.1016/j.carbpol.2004.04.020

Bizzini, A., Zhao, C., Budin-Verneuil, A., Sauvageot, N., Giard, J. C., Auffray, Y., et al. (2010). Glycerol is metabolized in a complex and strain-dependent 
manner in Enterococcus faecalis. J. Bacteriol. 192, 779-785. doi: 10.1128/ JB.00959-09

Bradbeer, C. (1965). The Clostridial fermentations of choline ethanolamine. J. Bacteriol. Chem. 240, 4669-4674.

Branca, F., Lartey, A., Oenema, S., Aguayo, V., Stordalen, G. A., Richardson, R., et al. (2019). Transforming the food system to fight non-communicable diseases. BMJ 365:1296. doi: 10.1136/bmj.1296

Broussard, J. L., and Devkota, S. (2016). The changing microbial landscape of Western society: diet, dwellings and discordance. Mol. Metab. 5, 737-742. doi: 10.1016/j.molmet.2016.07.007

Bryan, N. S., Alexander, D. D., Coughlin, J. R., Milkowski, A. L., and Boffetta, P. (2012). Ingested nitrate and nitrite and stomach cancer risk: an updated review. Food Chem. Toxicol. 50, 3646-3665. doi: 10.1016/j.fct.2012.07.062

Buysschaert, B., Kerckhof, F. M., Vandamme, P., De Baets, B., and Boon, N. (2018). Flow cytometric fingerprinting for microbial strain discrimination and physiological characterization. Cytometry A 93, 201-212. doi: 10.1002/cyto.a.23302

Cameotra, S. S., and Makkar, R. S. (2004). Recent applications of biosurfactants as biological and immunological molecules. Curr. Opin. Microbiol. 7, 262-266. doi: 10.1016/j.mib.2004.04.006

Canani, R. B., di Costanzo, M., Leone, L., Pedata, M., Meli, R., and Calignano, A. (2011). Potential beneficial effects of butyrate in intestinal and extraintestinal diseases. World J. Gastroenterol. 17, 1519-1528. doi: 10.3748/wjg.v17.i12.1519

Cani, P. D. (2017). Gut cell metabolism shapes the microbiome. Microbiome 357, 548-549. doi: 10.1126/science.aao2202

Carocho, M., Barreiro, M. F., Morales, P., and Ferreira, I. C. F. R. (2014). Adding molecules to food, pros and cons: a review on synthetic and natural food additives. Compr. Rev. Food Sci. Food Saf. 13, 377-399. doi: 10.1111/1541-4337.12065

Chang, Y. J., Pukall, R., Saunders, E., Lapidus, A., Copeland, A., Nolan, M., et al. (2010). Complete genome sequence of acidaminococcus fermentans type strain $\left(\mathrm{VR}_{4}\right)$. Stand. Genomic Sci. 3, 1-14. doi: 10.4056/sigs.1002553

Chassaing, B., Koren, O., Goodrich, J. K., Poole, A. C., Srinivasan, S., Ley, R. E., et al. (2015). Dietary emulsifiers impact the mouse gut microbiota promoting colitis and metabolic syndrome. Nature 519, 92-96. doi: 10.1038/nature14232

Chassaing, B., Van De Wiele, T., De Bodt, J., Marzorati, M., and Gewirtz, A. T. (2017). Dietary emulsifiers directly alter human microbiota composition and gene expression ex vivo potentiating intestinal inflammation. Gut 66, 1414-1427. doi: 10.1136/gutjnl-2016-313099

Costa, C. S., Del-ponte, B., Cecília, M., Assunção, F., and Santos, I. S. (2017). Consumption of ultra-processed foods and body fat during childhood and adolescence: a systematic review. 21, 148-159. doi: 10.1017/\$1368980017001331,

Daverey, A., and Pakshirajan, K. (2010). Sophorolipids from Candida bombicola using mixed hydrophilic substrates: production, purification and characterization. Colloids Surf. B: Biointerfaces 79, 246-253. doi: 10.1016/j. colsurfb.2010.04.002

De Boever, P., Deplancke, B., and Verstraete, W. (2000). Fermentation by gut microbiota cultured in a simulator of the human intestinal microbial ecosystem is improved by supplementing a soygerm powder. J. Nutr. 130, 2599-2606. doi: $10.1093 / \mathrm{jn} / 130.10 .2599$

Del Dot, T., Osawa, R., and Stackebrandt, E. (1993). Phascolarctobacterium faecium gen. nov, spec. nov., a novel taxon of the sporomusa group of bacteria. Syst. Appl. Microbiol. 16, 380-384. doi: 10.1016/S0723-2020(11) 80269-9

De Paepe, K., Kerckhof, F. M., Verspreet, J., Courtin, C. M., and Van de Wiele, T. (2017). Inter-individual differences determine the outcome of wheat bran colonization by the human gut microbiome. Environ. Microbiol. 19, 3251-3267. doi: 10.1111/1462-2920.13819

De Paepe, K., Verspreet, J., Verbeke, K., Raes, J., Courtin, C. M., and Van de Wiele, T. (2018). Introducing insoluble wheat bran as a gut microbiota niche in an in vitro dynamic gut model stimulates propionate and butyrate production and induces colon region specific shifts in the luminal and mucosal microbial community. Environ. Microbiol. 20, 3406-3426. doi: $10.1111 / 1462-2920.14381$

Deschasaux, M., Bouter, K., Prodan, A., Levin, E., Groen, A., Herrema, H., et al. (2019). Differences in gut microbiota composition in metabolic syndrome and type 2 diabetes subjects in a multi-ethnic population: the HELIUS study. Available at: https://app.oxfordabstracts.com/events/696/program-app/ submission/126560 (Accessed July 1, 2020).

Develter, D. W. G., and Lauryssen, L. M. L. (2010). Properties and industrial applications of sophorolipids. Eur. J. Lipid Sci. Technol. 112, 628-638. doi: 10.1002/ejlt.200900153
De Weirdt, R., Possemiers, S., Vermeulen, G., Moerdijk-Poortvliet, T. C. W., Boschker, H. T. S., Verstraete, W., et al. (2010). Human faecal microbiota display variable patterns of glycerol metabolism. FEMS Microbiol. Ecol. 74, 601-611. doi: 10.1111/j.1574-6941.2010.00974.x

Diether, N. E., and Willing, B. P. (2019). Microbial fermentation of dietary protein: an important factor in diet-microbe-host interaction. Microorganisms 7:19. doi: 10.3390/microorganisms7010019

Ding, R. X., Goh, W. R., Wu, R. N., Yue, X. Q., Luo, X., Khine, W. W. T., et al. (2019). Revisit gut microbiota and its impact on human health and disease. J. Food Drug Anal. 27, 623-631. doi: 10.1016/j.jfda.2018. 12.012

EFSA (2019). Europeans on today's food issues: new EU-wide survey comes out on first World Food Safety Day. Available at: https://www.efsa.europa. eu/en/press/news/190607 (Accessed February 23, 2020).

Ehehalt, R., Braun, A., Karner, M., Füllekrug, J., and Stremmel, W. (2010). Phosphatidylcholine as a constituent in the colonic mucosal barrierphysiological and clinical relevance. Biochim. Biophys. Acta 1801, 983-993. doi: 10.1016/j.bbalip.2010.05.014

European Commision (2011). 00172-Polysorbates-E433. Available at: https:// webgate.ec.europa.eu/foods_system/main/index.cfm? event=substance. view\&identifier=172 (Accessed August 6, 2019).

European Commision (2014). 00191-Sodium carboxymethyl cellulose-E466. Available at: https://webgate.ec.europa.eu/foods_system/main/index.cfm?event= substance.view\&identifier=191 (Accessed July 8, 2020).

European Commision (2018). 00115-Soy lecithin-E322. Available at: https:// webgate.ec.europa.eu/foods_system/main/index.cfm? event=substance. view\&identifier=115 (Accessed July 5, 2020).

Falcioni, T., Papa, S., and Gasol, J. M. (2008). Evaluating the flow-cytometric nucleic acid double-staining protocol in realistic situations of planktonic bacterial death. Appl. Environ. Microbiol. 74, 1767-1779. doi: 10.1128/ AEM.01668-07

FDA (2019a). CFR-Code of Federal Regulations Title 21-polysorbate 80. Available at: https://www.accessdata.fda.gov/scripts/cdrh/cfdocs/cfCFR/CFRSearch. cfm?fr=172.840 (Accessed July 5, 2020).

FDA (2019b). CFR-Code of Federal Regulations Title 21-sodium carboxymethyl cellulose. Available at: https:/www.accessdata.fda.gov/scripts/cdrh/cfdocs/ cfCFR/CFRSearch.cfm?fr=182.1745 (Accessed July 8, 2020).

FDA (2019c). CFR-Code of Federal Regulations Title 21-Soy lecithin. Available at: https://www.accessdata.fda.gov/scripts/cdrh/cfdocs/cfCFR/CFRSearch. cfm?fr=184.1400 (Accessed July 6, 2020).

FDA (2020). CARBOXYMETHYL CELLULOSE, SODIUM SALT. Available at: https://www.accessdata.fda.gov/scripts/fdcc/?set=FoodSubstances\&id=CARB OXYMETHYLCELLULOSESODIUMSALT\&sort=Sortterm\&order=ASC\&star trow=1\&type=basic\&search=carboxymethyl (Accessed July 5, 2020).

Fei, N., and Zhao, L. (2013). An opportunistic pathogen isolated from the gut of an obese human causes obesity in germfree mice. ISME J. 7, 880-884. doi: 10.1038 /ismej.2012

FMI (2020). Polysorbate-80 Market: Global Industry Analysis and Opportunity Assessment 2016-2026. Available at: https://www.futuremarketinsights.com/ reports/polysorbate-80-market

Francino, M. P. (2016). Antibiotics and the human gut microbiome: dysbioses and accumulation of resistances. Front. Microbiol. 6:1543. doi: 10.3389/ fmicb. 2015.01543

Girón, J. A. (1995). Expression of flagella and motility by Shigella. Mol. Biol. 18, 63-75. doi: 10.1111/j.1365-2958.1995.mmi_18010063.x

Guirro, M., Costa, A., Gual-Grau, A., Herrero, P., Torrell, H., Canela, N., et al. (2019). Effects from diet-induced gut microbiota dysbiosis and obesity can be ameliorated by fecal microbiota transplantation: a multiomics approach. PLoS One 14:e0218143. doi: 10.1371/journal.pone.0218143

Haba, E., Pinazo, A., Jauregui, O., Espuny, M. J., Infante, M. R., and Manresa, A. (2003). Physicochemical characterization and antimicrobial properties of rhamnolipids produced by Pseudomonas aeruginosa 47T2 NCBIM 40044. Biotechnol. Bioeng. 81, 316-322. doi: 10.1002/bit.10474

He, C., Cheng, D., Peng, C., Li, Y., Zhu, Y., and Lu, N. (2018). High-fat diet induces dysbiosis of gastric microbiota prior to gut microbiota in association with metabolic disorders in mice. Front. Microbiol. 9:639. doi: 10.3389/ fmicb.2018.00639

Hercules Inc., and Aqualon (1999). Aqualon-sodium carboxymethyl cellulosephysical and chemical propertie. Available at: https://www.academia. 
edu/8443565/Physical_and_Chemical_Properties_AQUALON_Sodium_ Carboxymethylcellulose (Accessed May 27, 2020).

Hörmann, B., Müller, M. M., Syldatk, C., and Hausmann, R. (2010). Rhamnolipid production by Burkholderia plantarii DSM 9509T. Eur. J. Lipid Sci. Technol. 112, 674-680. doi: 10.1002/ejlt.201000030

Hosseini, E., Grootaert, C., Verstraete, W., and Van de Wiele, T. (2011). Propionate as a health-promoting microbial metabolite in the human gut. Nutr. Rev. 69, 245-258. doi: 10.1111/j.1753-4887.2011.00388.x

Jiang, Z., Zhao, M., Zhang, H., Li, Y., Liu, M., and Feng, F. (2018). Antimicrobial emulsifier-glycerol monolaurate induces metabolic syndrome, gut microbiota dysbiosis, and systemic low-grade inflammation in low-fat diet fed mice. Mol. Nutr. Food Res. 62, 1-11. doi: 10.1002/mnfr.201700547

Jie, Z., Xia, H., Zhong, S. L., Feng, Q., Li, S., Liang, S., et al. (2017). The gut microbiome in atherosclerotic cardiovascular disease. Nat. Commun. 8:845. doi: 10.1038/s41467-017-00900-1

Joint FAO/WHO Expert Committee on Food Additives (1973). Toxicological evaluation of some food additives including anticaking agents, antimicrobials, antioxidants, emulsifiers and thickening agents. In IPCS Inchem. Available at: http://www.inchem.org/documents/jecfa/jecmono/v05je54.htm

Jones, M. N. (1999). Surfactants in membrane solubilisation. Int. J. Pharm. 177, 137-159. doi: 10.1016/s0378-5173(98)00345-7

Kaakoush, N. O. (2020). Sutterella species, IgA-degrading bacteria in ulcerative colitis. Trends Microbiol. 28, 519-522. doi: 10.1016/j.tim.2020.02.018

Kanehisa, M., Goto, S., Sato, Y., Furumichi, M., and Tanabe, M. (2012). KEGG for integration and interpretation of large-scale molecular data sets. Nucleic Acids Res. 40, 109-114. doi: 10.1093/nar/gkr988

Kim, H. S., Kim, Y. B., Lee, B. S., and Kim, E. K. (2005). Sophorolipid production by Candida bombicola ATCC 22214 from a corn-oil processing byproduct. J. Microbiol. Biotechnol. 15, 55-58.

Kozich, J. J., Westcott, S. L., Baxter, N. T., Highlander, S. K., and Schloss, P. D. (2013). Development of a dual-index sequencing strategy and curation pipeline for analyzing amplicon sequence data on the miseq illumina sequencing platform. Appl. Environ. Microbiol. 79, 5112-5120. doi: 10.1128/AEM.01043-13

Kramer, V., Nickerson, K. W., Hamlett, N. V., and O'Hara, C. (1984). Prevalence of extreme detergent resistance among the Enterobacteriaceae. Can. J. Microbiol. 30, 711-713. doi: $10.1139 / \mathrm{m} 84-106$

Langille, M. G. I., Zaneveld, J., Caporaso, J. G., McDonald, D., Knights, D., Reyes, J. A., et al. (2013). Predictive functional profiling of microbial communities using 16S rRNA marker gene sequences. Nat. Biotechnol. 31, 814-821. doi: 10.1038/nbt.2676

Le Chatelier, E., Nielsen, T., Qin, J., Prifti, E., Hildebrand, F., Falony, G., et al. (2013). Richness of human gut microbiome correlates with metabolic markers. Nature 500, 541-546. doi: 10.1038/nature12506

Lecomte, V., Kaakoush, N. O., Maloney, C. A., Raipuria, M., Huinao, K. D., Mitchell, H. M., et al. (2015). Changes in gut microbiota in rats fed a high fat diet correlate with obesity-associated metabolic parameters. PLoS One 10:e0126931. doi: 10.1371/journal.pone.0126931

Li, Z., Zhang, Y., Lin, J., Wang, W., and Li, S. (2019). High-yield di-rhamnolipid production by Pseudomonas aeruginosa YM4 and its potential application in MEOR. Molecules 24:1433. doi: 10.3390/molecules24071433

Liu, H., Wang, J., He, T., Becker, S., Zhang, G., Li, D., et al. (2018). Butyrate: a double-edged sword for health? Adv. Nutr. 9, 21-29. doi: 10.1093/advances/ nmx009

Lock, J. Y., Carlson, T. L., Wang, C. M., Chen, A., and Carrier, R. L. (2018). Acute exposure to commonly ingested emulsifiers alters intestinal mucus structure and transport properties. Sci. Rep. 8:10008. doi: 10.1038/s41598-018-27957-2

Louis, P., and Flint, H. J. (2017). Formation of propionate and butyrate by the human colonic microbiota. Environ. Microbiol. 19, 29-41. doi: $10.1111 / 1462-2920.13589$

Love, M. I., Huber, W., and Anders, S. (2014). Moderated estimation of fold change and dispersion for RNA-seq data with DESeq2. Genome Biol. 15:550. doi: 10.1186/s13059-014-0550-8

Luedde, M., Winkler, T., Heinsen, F. A., Rühlemann, M. C., Spehlmann, M. E., Bajrovic, A., et al. (2017). Heart failure is associated with depletion of core intestinal microbiota. ESC Heart Fail. 4, 282-290. doi: 10.1002/ ehf2.12155

Lupp, C., Robertson, M. L., Wickham, M. E., Sekirov, I., Champion, O. L., Gaynor, E. C., et al. (2007). Host-mediated inflammation disrupts the intestinal microbiota and promotes the overgrowth of Enterobacteriaceae. Cell Host Microbe 2, 119-129. doi: 10.1016/j.chom.2007.06.010

Ma, X., Li, H., and Song, X. (2012). Surface and biological activity of sophorolipid molecules produced by Wickerhamiella domercqiae var. sophorolipid CGMCC 1576. J. Colloid Interface Sci. 376, 165-172. doi: 10.1016/j.jcis.2012.03.007

Mallet, C. P. (ed.) (1992). Frozen food technology (Blackie Academic and Professional, an imprint of Chapman and Hall).

Martínez-del Campo, A., Bodea, S., Hamer, H. A., Marks, J. A., Haiser, H. J., Turnbaugh, P. J., et al. (2015). Characterization and detection of a widely distributed gene cluster that predicts anaerobic choline utilization by human gut bacteria. mBio 6, e00042-e00115. doi: 10.1128/mBio. 00042-15

Martínez Steele, E., Popkin, B. M., Swinburn, B., and Monteiro, C. A. (2017). The share of ultra-processed foods and the overall nutritional quality of diets in the US: evidence from a nationally representative cross-sectional study. Popul. Health Metrics 15:6. doi: 10.1186/s12963-017-0119-3

McMurdie, P. J., and Holmes, S. (2014). Waste not, want not: why rarefying microbiome data is inadmissible. PLoS Comput. Biol. 10:e1003531. doi: 10.1371/journal.pcbi.1003531

Michor, E. L., and Berg, J. C. (2015). Temperature effects on micelle formation and particle charging with span surfactants in apolar media. Langmuir 31, 9602-9607. doi: 10.1021/acs.langmuir.5b02711

Miclotte, L., and Van de Wiele, T. (2019). Food processing, gut microbiota and the globesity problem. Crit. Rev. Food Sci. Nutr. 60, 1769-1782. doi: 10.1080/10408398.2019.1596878

Mittal, N., Budrene, E. O., Brenner, M. P., and Van Oudenaarden, A. (2003). Motility of Escherichia coli cells in clusters formed by chemotactic aggregation. Proc. Natl. Acad. Sci. U. S. A. 100, 13259-13263. doi: 10.1073/pnas.22 33626100

Monteiro, C. A., Cannon, G., Moubarac, J., Levy, R. B., Louzada, M. L. C., and Jaime, P. C. (2017). Commentary the UN decade of nutrition, the NOVA food classi fi cation and the trouble with ultra-processing. Public Health Nutr. 21, 5-17. doi: 10.1017/S1368980017000234

Monteiro, C. A., Moubarac, J. -C., Cannon, G., Ng, S. W., and Popkin, B. (2013). Ultra-processed products are becoming dominant in the global food system. Obes. Rev. 14, 21-28. doi: 10.1111/obr.12107

Moon, C., Baldridge, M. T., Wallace, M. A., Burnham, C. A. D., Virgin, H. W., and Stappenbeck, T. S. (2015). Vertically transmitted faecal IgA levels determine extra-chromosomal phenotypic variation. Nature 521, 90-93. doi: 10.1038/nature14139

Moore, S. L. (1997). The Mechanisms of Antibacterial Action of Some Nonionic Surfactants. University of Brighton in collaboration with Unilever Research.

Mortensen, A., Aguilar, F., Crebelli, R., Di Domenico, A., Frutos, M. J., Galtier, P., et al. (2017). Re-evaluation of lecithins (E 322) as a food additive. EFSA J. 15:e04742. doi: 10.2903/j.efsa.2017.4742

Mourad, A. M., De Carvalho Pincinato, E., Mazzola, P. G., Sabha, M., and Moriel, P. (2010). Influence of soy lecithin administration on hypercholesterolemia. Cholesterol 2010:824813. doi: 10.1155/2010/824813

Moussa, T. A. A., Mohamed, M. S., and Samak, N. (2014). Production and characterization of di-rhamnolipid produced by Pseudomonas aeruginosa TMN. Braz. J. Chem. Eng. 31, 867-880. doi: 10.1590/0104-6632.201403 $14 \mathrm{~s} 00002473$

Msagati, T. A. (2012). "Chemistry of food additives and preservatives" in Chemistry of food additives and preservatives. Wiley-Blackwell, 33-62.

Mu, C., Yang, Y., Luo, Z., Guan, L., and Zhu, W. (2016). The colonic microbiome and epithelial transcriptome are altered in rats fed a high-protein diet compared with a normal-protein diet. J. Nutr. 146, 474-483. doi: 10.3945/ jn. 115.223990

Muleviciene, A., D’Amico, F., Turroni, S., Candela, M., and Jankauskiene, A. (2018). Iron deficiency anemia-related gut microbiota dysbiosis in infants and young children: a pilot study. Acta Microbiol. Immunol. Hung. 65, 551-564. doi: 10.1556/030.65.2018.045

Musso, G., Gambino, R., and Cassader, M. (2010). Obesity, diabetes, and gut microbiota: the hygiene hypothesis expanded? Diabetes Care 33, 2277-2284. doi: $10.2337 / \mathrm{dc} 10-0556$

Naso, J. N., Bellesi, F. A., Ruiz-Henestrosa, V. M. R., and Pilosof, A. M. R. (2019). Studies on the interactions between bile salts and food emulsifiers under in vitro duodenal digestion conditions to evaluate their bile salt 
binding potential. Colloids Surf. B: Biointerfaces 174, 493-500. doi: 10.1016/j. colsurfb.2018.11.024

Nickerson, K. W., and Aspedon, A. (1992). Detergent-shock response in enteric bacteria. Mol. Biol. 6, 957-961. doi: 10.1111/j.1365-2958.1992. tb02161.x

Nielsen, C. K., Kjems, J., Mygind, T., Snabe, T., and Meyer, R. L. (2016). Effects of tween 80 on growth and biofilm formation in laboratory media. Front. Microbiol. 7:1878. doi: 10.3389/fmicb.2016.01878

Nitschke, M., and Silva, S. S. E. (2018). Recent food applications of microbial surfactants. Crit. Rev. Food Sci. Nutr. 58, 631-638. doi: 10.1080/1040839 8.2016.1208635

Oksanen, J. F., Blanchet, G., Friendly, M., Kindt, R., Legendre, P., McGlinn, D., et al. (2019). Vegan: community ecology package. R package version 2.5-6. Available at: https://cran.r-project.org/web/packages/vegan/index. html

Otto, R. T., Daniel, H. -J., Pekin, G., Müller-Decker, K., Fürstenberger, G., Reuss, M., et al. (1999). Production of sophorolipids from whey. Appl. Microbiol. Biotechnol. 52, 495-501. doi: 10.1007/s002530051551

Parreidt, T. S., Schott, M., Schmid, M., and Müller, K. (2018). Effect of presence and concentration of plasticizers, vegetable oils, and surfactants on the properties of sodium-alginate-based edible coatings. Int. J. Mol. Sci. 19:742. doi: 10.3390/ijms19030742

Patterson, E. E., Ryan, P. M., Cryan, J. F., Dinan, T. G., Paul Ross, R., Fitzgerald, G. F., et al. (2016). Gut microbiota, obesity and diabetes. Postgrad. Med. J. 92, 286-300. doi: 10.1136/postgradmedj-2015-133285

Payne, A. N., Chassard, C., and Lacroix, C. (2012). Gut microbial adaptation to dietary consumption of fructose, artificial sweeteners and sugar alcohols: implications for host-microbe interactions contributing to obesity. Obes. Rev. 13, 799-809. doi: 10.1111/j.1467-789X.2012.01009.x

Pedersen, C., Ijaz, U. Z., Gallagher, E., Horton, F., Ellis, R. J., Jaiyeola, E., et al. (2018). Fecal Enterobacteriales enrichment is associated with increased in vivo intestinal permeability in humans. Physiol. Rep. 6:e13649. doi: 10.14814/ phy2.13649

Pogorzelski, S., Watrobska-Swietlikowska, D., and Sznitowska, M. (2012). Surface tensometry studies on formulations of surfactants with preservatives as a tool for antimicrobial drug protection characterization. J. Biophys. Chem. 3, 324-333. doi: $10.4236 / \mathrm{jbpc} .2012 .34040$

Props, R., Monsieurs, P., Mysara, M., Clement, L., and Boon, N. (2016). Measuring the biodiversity of microbial communities by flow cytometry. Methods Ecol. Evol. 7, 1376-1385. doi: 10.1111/2041-210X.12607

Quast, C., Pruesse, E., Yilmaz, P., Gerken, J., Schweer, T., Yarza, P., et al. (2013). The SILVA ribosomal RNA gene database project: improved data processing and web-based tools. Nucleic Acids Res. 41, D590-D596. doi: 10.1093/nar/gks1219

Rajagopal, S., Eis, N., Bhattacharya, M., and Nickerson, K. W. (2003). Membranederived oligosaccharides (MDOs) are essential for sodium dodecyl sulfate resistance in Escherichia coli. FEMS Microbiol. Lett. 223, 25-31. doi: 10.1016/ S0378-1097(03)00323-9

Rajagopal, S., Sudarsan, N., and Nickerson, K. W. (2002). Sodium dodecyl sulfate hypersensitivity of clpP and clpB mutants of Escherichia coli. Appl. Environ. Microbiol. 68, 4117-4121. doi: 10.1128/aem.68.8.4117-4121. 2002

Ramos, H. C., Rumbo, M., and Sirard, J. (2004). Bacterial flagellins: mediators of pathogenicity and host immune responses in mucosa. Trends Microbiol. 12, 510-517. doi: 10.1016/j.tim.2004.09.002

Rauber, F., da Costa Louzada, M. L., Steele, E. M., Millett, C., Monteiro, C. A., and Levy, R. B. (2018). Ultra-processed food consumption and chronic non-communicable diseases-related dietary nutrient profile in the UK (2008-2014). Nutrients 10:587. doi: 10.3390/nu10050587

R Core Team (2016). R: A Language and Environment for Statistical Computing. R Foundation for Statistical Computing. Available at: https://www.R-project. org

Reichardt, N., Duncan, S. H., Young, P., Belenguer, A., McWilliam Leitch, C., Scott, K. P., et al. (2014). Phylogenetic distribution of three pathways for propionate production within the human gut microbiota. ISME J. 8, 1323-1335. doi: 10.1038/ismej.2014.14

Rodionova, I. A., Li, X., Thiel, V., Stolyar, S., Stanton, K., Fredrickson, J. K., et al. (2013). Comparative genomics and functional analysis of rhamnose catabolic pathways and regulons in bacteria. Front. Microbiol. 4:407. doi: 10.3389/fmicb.2013.00407

Shin, N. R., Whon, T. W., and Bae, J. W. (2015). Proteobacteria: microbial signature of dysbiosis in gut microbiota. Trends Biotechnol. 33, 496-503. doi: 10.1016/j.tibtech.2015.06.011

Stremmel, W., Robert, A. H., Karner, E. M., and Braun, A. (2010). Phosphatidylcholine (lecithin) and the mucus layer: evidence of therapeutic efficacy in ulcerative colitis? Dig. Dis. 28, 490-496. doi: 10.1159/000320407

Szymczyk, K., Zdziennicka, A., and Jańczuk, B. (2018). Adsorption and aggregation properties of some polysorbates at different temperatures. J. Solut. Chem. 47, 1824-1840. doi: 10.1007/s10953-018-0823-Z

Tang, W. H. W., and Hazen, S. L. (2014). The contributory role of gut microbiota in cardiovascular disease find the latest version: the contributory role of gut microbiota in cardiovascular disease. J. Clin. Investig. 124, 4204-4211. doi: 10.1172/JCI72331

Tito, R. Y., Cypers, H., Joossens, M., Varkas, G., Van Praet, L., Glorieus, E., et al. (2017). Brief report: dialister as a microbial marker of disease activity in spondyloarthritis. Arthritis Rheum. 69, 114-121. doi: 10.1002/ art.39802

Tominaga, A., Mahmoud, M. A. H., Al Mamun, A. A. M., and Mukaihara, T. (2001). Characterization of cryptic flagellin genes in Shigella boydii and Shigella dysenteriae. Genes Genet. Syst. 76, 111-120. doi: 10.1266/ggs.76.111

Turnbaugh, P. J., Hamady, M., Yatsunenko, T., Cantarel, B. L., Duncan, A., Ley, R. E., et al. (2009). A core gut microbiome in obese and lean twins. Nature 457, 480-484. doi: 10.1038/nature07540

Van Bogaert, I. (2008). Microbial synthesis of sophorolipids by the yeast candida bombicola. Ghent University.

Van Bogaert, I., Zhang, J., and Soetaert, W. (2011). Microbial synthesis of sophorolipids. Process Biochem. 46, 821-833. doi: 10.1016/j.procbio.2011.01.010

Vandeputte, D., Kathagen, G., D’Hoe, K., Vieira-Silva, S., Valles-Colomer, M., Sabino, J., et al. (2017). Quantitative microbiome profiling links gut community variation to microbial load. Nature 551, 507-511. doi: 10.1038/ nature 24460

Van Haesendonck, I., and Vanzeveren, E. C. A. (2006). (12) Patent Application Publication (10) Pub. No.: US 2006/0233935 A1.1(19).

Van Nevel, S., Koetzsch, S., Weilenmann, H. U., Boon, N., and Hammes, F. (2013). Routine bacterial analysis with automated flow cytometry. J. Microbiol. Methods 94, 73-76. doi: 10.1016/j.mimet.2013.05.007

Verhoog, S., Taneri, P. E., Díaz, Z. M. R., Marques-Vidal, P., Troup, J. P., Bally, L., et al. (2019). Dietary factors and modulation of bacteria strains of Akkermansia muciniphila and faecalibacterium prausnitzii: a systematic review. Nutrients 11:1565. doi: 10.3390/nu11071565

Vilchez-Vargas, R., Geffers, R., Suárez-Diez, M., Conte, I., Waliczek, A., Kaser, V. S., et al. (2013). Analysis of the microbial gene landscape and transcriptome for aromatic pollutants and alkane degradation using a novel internally calibrated microarray system. Environ. Microbiol. 15, 1016-1039. doi: 10.1111/j. 1462-2920.2012.02752.x

Vu, K. A., Tawfiq, K., and Chen, G. (2015). Rhamnolipid transport in biocharamended agricultural soil. Water Air Soil Pollut. 226, 1-8. doi: 10.1007/ s11270-015-2497-0

Wang, L., Christophersen, C. T., Sorich, M. J., Gerber, J. P., Angley, M. T., and Conlon, M. A. (2013). Increased abundance of Sutterella spp. and Ruminococcus torques in feces of children with autism spectrum disorder. Mol. Autism 4:42. doi: 10.1186/2040-2392-4-42

Wang, Z., Klipfell, E., Bennett, B. J., Koeth, R., Levison, B. S., Dugar, B., et al. (2011). Gut flora metabolism of phosphatidylcholine promotes cardiovascular disease. Nature 472, 57-65. doi: 10.1038/nature09922

Ward, T., Larson, J., Meulemans, J., Hillman, B., Lynch, J., Sidiripoulos, D., et al. (2017). BugBase predicts organism-level microbiome phenotypes. bioRxiv [preprint]. doi: $10.1101 / 133462$

Waterlander, W. E., Ni Mhurchu, C., Eyles, H., Vandevijvere, S., Cleghorn, C., Scarborough, P., et al. (2018). Food futures: developing effective food systems interventions to improve public health nutrition. Agric. Syst. 160, 124-131. doi: 10.1016/j.agsy.2017.01.006

Weitkunat, K., Schumann, S., Nickel, D., Kappo, K. A., Petzke, K. J., Kipp, A. P., et al. (2016). Importance of propionate for the repression of hepatic lipogenesis and improvement of insulin sensitivity in high-fat diet-induced obesity. Mol. Nutr. Food Res. 60, 2611-2621. doi: 10.1002/mnfr.201600305 
Wlodkovic, D., Skommer, J., and Darzynkiewicz, Z. (2009). Flow cytometrybased apoptosis detection Donald. Mol. Biol. 559, 313-332. doi: 10.1007/978-1-60327-017-5

$\mathrm{Wu}, \mathrm{Y}$., and Wang, T. (2003). Soybean lecithin fractionation and functionality. J. Am. Oil Chem. Soc. 80, 319-326. doi: 10.1007/s11746-003-0697-x

Yao, C. K., Muir, J. G., and Gibson, P. R. (2016). Review article: insights into colonic protein fermentation, its modulation and potential health implications. Aliment. Pharmacol. Ther. 43, 181-196. doi: 10.1111/apt.13456

Zhang, H., Dudley, E. G., and Harte, F. (2017). Critical synergistic concentration of lecithin phospholipids improves the antimicrobial activity of eugenol against Escherichia coli. Appl. Environ. Microbiol. 83, 1-9. doi: 10.1128/ AEM.01583-17
Conflict of Interest: The authors declare that the research was conducted in the absence of any commercial or financial relationships that could be construed as a potential conflict of interest.

Copyright (C) 2020 Miclotte, De Paepe, Rymenans, Callewaert, Raes, Rajkovic, Van Camp and Van de Wiele. This is an open-access article distributed under the terms of the Creative Commons Attribution License (CC BY). The use, distribution or reproduction in other forums is permitted, provided the original author(s) and the copyright owner(s) are credited and that the original publication in this journal is cited, in accordance with accepted academic practice. No use, distribution or reproduction is permitted which does not comply with these terms. 